\title{
A global shallow water model using high order multi-moment constrained finite volume method and icosahedral grid
}

\author{
Satoshi Ii ${ }^{\mathrm{a}}$, Feng Xiao ${ }^{\mathrm{b}, \mathrm{c}, *}$ \\ a Department of Mechanical Engineering, The University of Tokyo, 7-3-1 Hongo Bunkyo-ku, Tokyo 113-8656, Japan \\ ${ }^{\mathrm{b}}$ Department of Energy Sciences, Tokyo Institute of Technology, 4259 Nagatsuta, Midori-ku, Yokohama 226-8502, Japan \\ ${ }^{\mathrm{c}}$ LHD, Institute of Mechanics, Chinese Academy of Sciences, 15 Beisihuanxi Road, Beijing 100080, China
}

\section{A R T I C L E I N F O}

\section{Article history:}

Received 9 June 2009

Received in revised form 24 October 2009

Accepted 6 November 2009

Available online 17 November 2009

\section{Keywords:}

Global shallow water model

Icosahedral grid

High order accuracy

Finite volume method

Multi-moment

Compact-stencil

\begin{abstract}
A B S T R A C T
A novel accurate numerical model for shallow water equations on sphere have been developed by implementing the high order multi-moment constrained finite volume (MCV) method on the icosahedral geodesic grid. High order reconstructions are conducted cellwisely by making use of the point values as the unknowns distributed within each triangular cell element. The time evolution equations to update the unknowns are derived from a set of constrained conditions for two types of moments, i.e. the point values on the cell boundary edges and the cell-integrated average. The numerical conservation is rigorously guaranteed. In the present model, all unknowns or computational variables are point values and no numerical quadrature is involved, which particularly benefits the computational accuracy and efficiency in handling the spherical geometry, such as coordinate transformation and curved surface.

Numerical formulations of third and fourth order accuracy are presented in detail. The proposed numerical model has been validated by widely used benchmark tests and competitive results are obtained. The present numerical framework provides a promising and practical base for further development of atmospheric and oceanic general circulation models.
\end{abstract}

(c) 2009 Elsevier Inc. All rights reserved.

\section{Introduction}

One of the crucial issues in developing numerical models for global circulations of atmosphere and ocean is how to accurately represent the discretized or numerical form of the governing equations in spherical geometry. The spectral model [5] makes use of the Fourier representation in the longitudinal direction and the Legendre representation in the latitudinal direction, and had found great success in the past. However, because of the global nature, the numerical models based on spectral transformation intrinsically suffer from poor computational efficiency when implemented on a massively parallel hardware platform and the spurious oscillations around discontinuities or steep gradients. More attention and efforts have been recently devoted to numerical methods with local representations over compact computational stencils [59].

Instead of the traditional latitude-longitude grid, the most natural grid to represent a spherical geometry, grids with more uniform grid spacing over the whole globe share an increasing popularity among the researchers. Two grids

\footnotetext{
* Corresponding author. Address: Department of Energy Sciences, Tokyo Institute of Technology, 4259 Nagatsuta, Midori-ku, Yokohama 226-8502, Japan. Tel./fax: +8610 82543877.

E-mail address: xiao@es.titech.ac.jp (F. Xiao).
} 
that are getting widely accepted and accommodate numerical conservation are the cubed-sphere grid and the icosahedral geodesic grid.

The cubed-sphere grid is generated by mapping the inscribed cube on to the surface of the globe which is then projected on six identical patches. Global models that use the cubed-sphere grid are found in $[44,27,28,36,41,30,31,42,33,4]$.

The icosahedral geodesic grid, on the other hand, starts from 20 triangles curving over the sphere. Refining the grid is then conducted by further dividing the triangles into sub-regions of triangle. This process can be repeated until the level of grid resolution is reached. The icosahedral geodesic grid is one that results in the most isotropic computational mesh elements over the whole sphere, and had been used as the base for dynamical cores in history $[43,56,57,26,6]$. Recent efforts from different groups have led to significant development of global models from shallow water model to atmospheric models $[48,38,14,54,15,39,25,55,16,17,49,45]$ using icosahedral geodesic grid. It should be also notified that projecting the equations in Cartesian coordinates onto 2D space on the surface of sphere is getting a popularity in the community $[23,2,11]$.

Another recent trend which is worthy of particular remarks in the development of the dynamical cores for global geophysical circulation models is the implementation of high order schemes with local reconstructions that have been proposed for more general applications in computational fluid dynamics. The numerical simulations of global circulations generally requires long-term computation, thus numerical conservation and computational efficiency are also demanded in addition to the accuracy. As the practice toward that direction, the discontinuous Galerkin (DG) method has been recently applied to the icosahedral geodesic grid by Giraldo et al. [15,17], and to the cubed-sphere grid by Nair et al. [30,31]. The DG method, originally introduced in [37] and developed by Cockburn and Shu [7-10] into a framework for solving non-linear time dependent hyperbolic conservation laws, defines the local degrees of freedom (DOF) on each element or cell as the unknowns, which are then updated by the Galerkin formulation with the approximate Riemann solver at the cell boundary. Thus, the DG method provides the local mass conservation, high order accuracy and flexibility due to the locally defined DOF. Ascribing the spectral convergence of the DG method, very high order accuracy was reported in [15,30,31]. The DG method, however, requires numerical quadrature intrinsically due to the Galerkin formulation, and is usually computationally expensive.

We have been recently working on developing a novel class of high order numerical formulations for computational fluid dynamics by using multi-moment concept [67,68,61,62,18,63,64,19,1,21,4]. The underlying idea is to make use of different kinds of discretized quantities, such as the point value, derivatives and cell-integrated value, which are collectively called moments in our context. With all the employed moments locally defined over each mesh cell, high order reconstructions can be built on very compact mesh stencil. The numerical formulations for the flux function and its spatial derivatives, which are required to update the moments, are then approximated with high order, provided the local multi-moment reconstructions are readily available. In a multi-moment method, different moments can be updated by different formulations, for example the point value can be updated by a point-wise Riemann solver and the cell-integrated average by a finite volume formulation that leads to the rigorous numerical conservation. We have reported two global multi-moment finite volume shallow water models by separately using the Yin-Yang over set grid [24] and the cubed-sphere grid [4].

An alternative to the multi-moment finite volume formulation, where the moments are directly used as the unknowns which need to be updated in time at every step, is to define the unknowns as the values at the points collocated within each grid cell. The resulting scheme is so-called the multi-moment constrained finite volume (MCV) method [20]. In an MCV method, the governing equations of the moments provide constraints which are then converted to the time evolution equations for the unknowns through an interpolation function. In practice, a multi-moment constrained Lagrange interpolation is used to link the moments and the point values defined within each single cell at equally spaced points as the unknowns. The finite volume constraint on the cell average exactly guarantees the numerical conservation. Shown in [20], the MCV method demonstrates spectral convergence behavior to both linear and non-linear problems. The major different between the MCV method and the previous multi-moment finite volume method is that the cell-integrated average is no longer a computational variable in the MCV method. All the computational variables are the point values, so, one do not need to calculate numerical quadrature. This is particularly beneficial when source term, metric term and irregular mesh are involved. The point-wise computation of the metric terms in the MCV formulation presented in this paper allows us to easily get high accuracy for unstructured and curved grid generated by either analytical formula or numerical means.

In this paper, the MCV method is applied to the global shallow water model using the icosahedral geodesic grid. The basic formulation is obtained by extending our previous work [20] to the triangular mesh. In addition, the local high order curvilinear element is employed to ensure the uniform convergence for high order schemes. The governing equations are described in the 3D Cartesian coordinate system with the restriction condition to remain the flow along the surface of the Earth [50]. It is quite suitable and easy to implement the MCV method in such a framework because all unknowns in the MCV method are the point values to which the computations of the restriction and source terms are carried out straightforwardly.

The rest of this paper consists of the following parts. Section 2 introduces the basic idea of presenting the global shallow water equations in the 3D Cartesian coordinate system. The MCV model on the spherical geometry is described in Section 3, where the third and fourth order formulations are discussed in detail. In Section 4, we verify our numerical model by numerical experiments including the standard test set for the global shallow water model proposed by Williamson et al. [58] and others. We also make a few comments on our practice of implementing multi-moment finite volume method in Yin-Yang overset grid and cubed-sphere grid. Finally, we end the paper with some conclusion remarks in Section 5. 


\section{Global shallow water model in the Cartesian coordinate system}

In this paper, we follow Swarztrauber et al. [50] and re-write the shallow water equations on the surface of the sphere as a three-dimensional system in Cartesian coordinates to avoid the pole singularity. A projection is then used to restrict the solutions on the surface of the sphere.

The global shallow water model in the three-dimensional Cartesian coordinate system is written as follows,

$$
Q_{t}+F(Q)_{x}+G(Q)_{y}+H(Q)_{z}=S,
$$

where

$$
Q=\left[\begin{array}{c}
h \\
h u \\
h v \\
h w
\end{array}\right], \quad F=\left[\begin{array}{c}
h u \\
h u^{2}+\frac{1}{2} g h^{2} \\
h u v \\
h u w
\end{array}\right], \quad G=\left[\begin{array}{c}
h v \\
h u v \\
h v^{2}+\frac{1}{2} g h^{2} \\
u v w
\end{array}\right], \quad H=\left[\begin{array}{c}
h w \\
h u w \\
h v w \\
h w^{2}+\frac{1}{2} g h^{2}
\end{array}\right], \quad S=\left[\begin{array}{c}
0 \\
f\left(\frac{z}{a} h v-\frac{y}{a} h w\right)-g h b_{x} \\
f\left(\frac{x}{a} h w-\frac{z}{a} h u\right)-g h b_{y} \\
f\left(\frac{y}{a} h u-\frac{x}{a} h v\right)-g h b_{z}
\end{array}\right],
$$

and

$$
f=\frac{2 \Omega z}{a} .
$$

In the above equations, $h$ denotes the height measured from the bed elevation $b, \mathbf{u}=(u, v, w)$ the velocity vector in the Cartesian coordinate system and $f$ the Coriolis parameter. The radius, gravity acceleration and angular velocity of the Earth are respectively specified as $a=6.37122 \times 10^{6}, g=9.80616$ and $\Omega=7.292 \times 10^{-5}$.

In the presence of the bottom topography, i.e. $b_{x} \neq 0$ or $b_{y} \neq 0$ or $b_{z} \neq 0$, spurious disturbance may arise if the numerical formulation for the flux function does not well balance that for the source term of bottom topography.

In case of the "still state" ( $h+b=$ constant and $\mathbf{u}=0),(2.1)$ reduces to following relationships,

$$
\frac{1}{2} g \frac{\partial h^{2}}{\partial x}=-g h \frac{\partial b}{\partial x}, \quad \frac{1}{2} g \frac{\partial h^{2}}{\partial y}=-g h \frac{\partial b}{\partial y}, \quad \frac{1}{2} g \frac{\partial h^{2}}{\partial z}=-g h \frac{\partial b}{\partial z} .
$$

It is obvious that the numerical discretization must satisfy the above relationships, i.e. the numerical flux has to be well-balanced with the computation of the topographic source term, to maintain the exact still state solution. It is the so-called Cproperty $[3,66]$. However, this is not automatically guaranteed, and an unbalanced discretization usually causes unphysical oscillations in numerical solution. We can easily circumvent this problem by recasting (2.2) in terms of the total height or the elevation of the water surface $T=h+b$, which yields another equivalent form of (2.1) by using the total height in flux and topographic source terms as,

$$
Q=\left[\begin{array}{c}
h \\
h u \\
h v \\
h w
\end{array}\right], \quad F=\left[\begin{array}{c}
h u \\
h u^{2}+\frac{1}{2} g T^{2} \\
h u v \\
h u w
\end{array}\right], \quad G=\left[\begin{array}{c}
h v \\
h u v \\
h v^{2}+\frac{1}{2} g T^{2} \\
u v w
\end{array}\right], \quad H=\left[\begin{array}{c}
h w \\
h u w \\
h v w \\
h w^{2}+\frac{1}{2} g T^{2}
\end{array}\right], \quad S=\left[\begin{array}{c}
0 \\
f\left(\frac{z}{a} h v-\frac{y}{a} h w\right)+g b T_{x} \\
f\left(\frac{x}{a} h w-\frac{z}{a} h u\right)+g b T_{y} \\
f\left(\frac{y}{a} h u-\frac{x}{a} h v\right)+g b T_{z}
\end{array}\right] .
$$

It is easy to verify that the C-property is satisfied if we use the form given by (2.4).

\subsection{High order treatment for the curved surface on the sphere}

In this paper, we make use of the icosahedral geodesic grid firstly proposed to solve the barotropic models by Sadourny et al. [43] and Williamson [56]. The grid is generated from the regular icosahedron (Fig. 1(a)). Shown in Fig. 1(b)-(d), refined grid is created by further dividing each triangle. So, the globe is finally covered by spherical triangular cells of required resolution, see details in Appendix A.

It is necessary to treat each triangular surface of the sphere as a curved surface to maintain the high order accuracy. In the present scheme, spatial discretization is carried out on the spherical triangle mesh cell. Considering a sphere centered at the origin of the Cartesian coordinates, we introduce the local coordinate system $(\xi, \eta, r)$ for each single cell, where $r$ indicates the radial coordinate and $\xi$ and $\eta$ span a local base on the surface of the sphere. Thus, the transformation of the global coordinates $(x, y, z)$ to the local coordinates $(\xi, \eta, r)$ can be expressed by scaling a position vector onto a mesh element on the spherical surface as $[47,14]$,

$$
\tilde{x}(\xi, \eta)=x(\xi, \eta) r ; \quad \tilde{y}(\xi, \eta)=y(\xi, \eta) r ; \quad \tilde{z}(\xi, \eta)=z(\xi, \eta) r .
$$

For a given radius $r=a$ we project the $R^{3}$ space of $(x, y, z)$ to $R^{2}$ space of $(\xi, \eta)$ that is normalized as the triangle $(0 \leqslant \xi, \eta \leqslant 1, \xi+\eta=1)$ shown in Fig. 2 . The Jacobian matrix is then written as, 


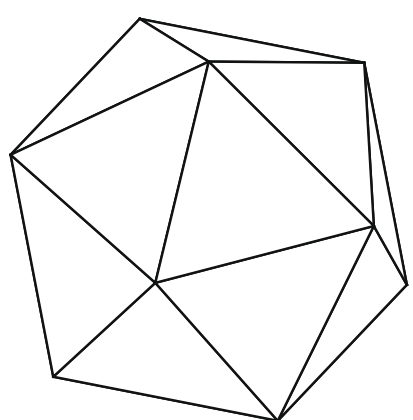

(a) Regular icosahedron

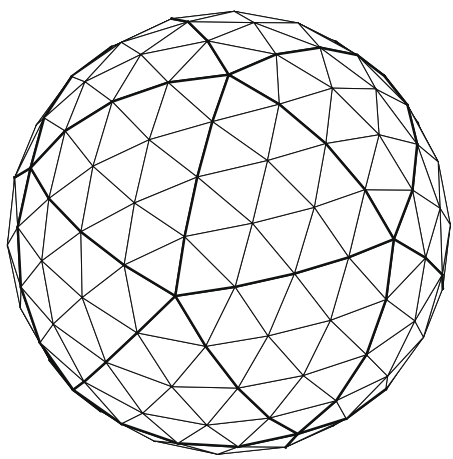

(c) $N=4$

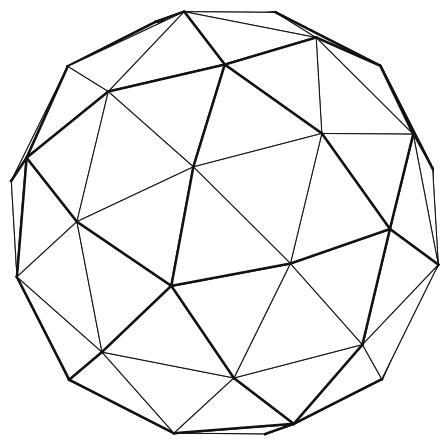

(b) $N=2$

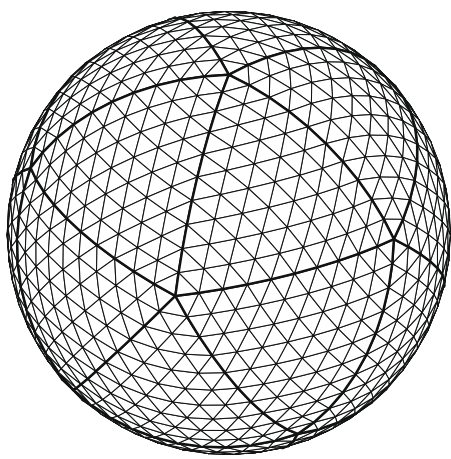

(d) $N=10$

Fig. 1. The gradual refinement of the icosahedral geodesic grids.

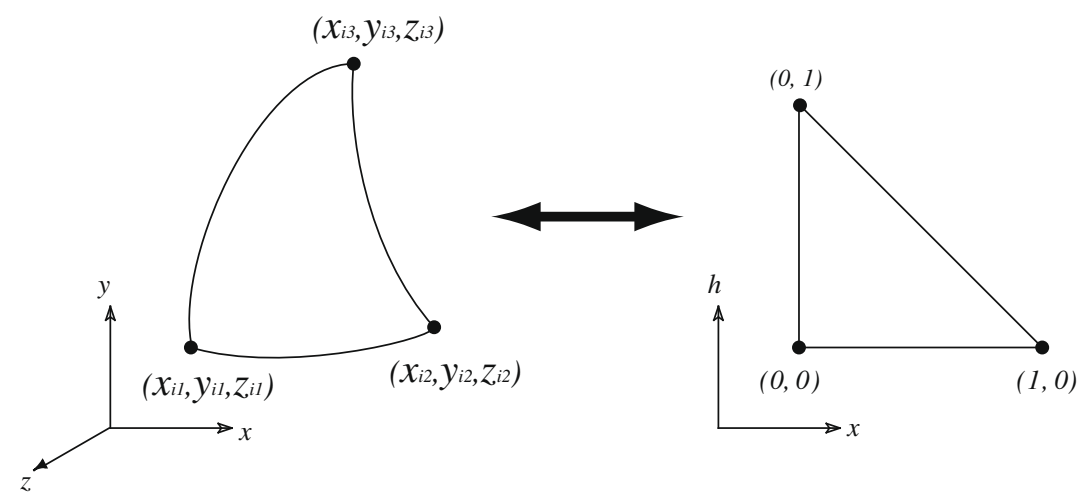

Fig. 2. Relationship between the global coordinate system and the local coordinate system.

$$
J(\xi, \eta)=\left[\begin{array}{ccc}
\frac{\partial \tilde{x}(\xi, \eta)}{\partial \xi} & \frac{\partial \tilde{x}(\xi, \eta)}{\partial \eta} & \frac{\tilde{x}(\xi, \eta)}{a} \\
\frac{\partial \tilde{y}(\xi, \eta)}{\partial \xi} & \frac{\partial \tilde{y}(\xi, \eta)}{\partial \eta} & \frac{\tilde{y}(\xi, \eta)}{a} \\
\frac{\partial \tilde{z}(\xi, \eta)}{\partial \xi} & \frac{\partial \tilde{z}(\xi, \eta)}{\partial \eta} & \frac{\tilde{z}(\xi, \eta)}{a}
\end{array}\right]=\left[\begin{array}{ccc}
\tilde{x}_{\xi}(\xi, \eta) & \tilde{x}_{\eta}(\xi, \eta) & \tilde{x}(\xi, \eta) / a \\
\tilde{y}_{\xi}(\xi, \eta) & \tilde{y}_{\eta}(\xi, \eta) & \tilde{y}(\xi, \eta) / a \\
\tilde{z}_{\xi}(\xi, \eta) & \tilde{z}_{\eta}(\xi, \eta) & \tilde{z}(\xi, \eta) / a
\end{array}\right] .
$$

Shown later, a scheme of $L$ th order accuracy which requires a $(L-1)$ th order basis function with at least $K=L(L+1) / 2$ constrained conditions at collocation points within each cell. Given $K$ collocation points, $\mathbf{p}_{i l}=\left(x_{i l}, y_{i l}, z_{i l}\right)(l=1, \ldots, K)$, over cell $s_{i}$, the coordinates of any point $\left(X_{i}, Y_{i}, Z_{i}\right)$ within $s_{i}$ can be expressed in terms of the local coordinate system $(\xi, \eta)$ by

$$
X_{i}(\xi, \eta)=\sum_{l=1}^{K} c_{i l}(\xi, \eta) x_{i l}, \quad Y_{i}(\xi, \eta)=\sum_{l=1}^{K} c_{i l}(\xi, \eta) y_{i l}, \quad Z_{i}(\xi, \eta)=\sum_{l=1}^{K} c_{i l}(\xi, \eta) z_{i l},
$$


where $c_{i l}(\xi, \eta)(l=1, \ldots, K)$ is the basis function of the Lagrange polynomial. Similarly, given the physical quantity $Q$ at the collocation points $\left(Q_{i l} ;(l=1, \ldots, K)\right)$, the interpolation reconstruction is given by

$$
P_{i}(Q: \xi, \eta)=\sum_{l=1}^{K} c_{i l}(\xi, \eta) Q_{i l} .
$$

The Jacobian transformation matrix is then written as,

$$
J_{i}(\xi, \eta)=\left[\begin{array}{ccc}
\frac{\partial X_{i}(\xi, \eta)}{\partial \xi} & \frac{\partial X_{i}(\xi, \eta)}{\partial \eta} & \frac{X_{i}(\xi, \eta)}{a} \\
\frac{\partial Y_{i}(\xi, \eta)}{\partial \xi} & \frac{\partial Y_{i}(\xi, \eta)}{\partial \eta} & \frac{Y_{i}(\xi, \eta)}{a} \\
\frac{\partial Z_{i}(\xi, \eta)}{\partial \xi} & \frac{\partial Z_{i}(\xi, \eta)}{\partial \eta} & \frac{Z_{i}(\xi, \eta)}{a}
\end{array}\right]=\left[\begin{array}{ccc}
X_{\xi i}(\xi, \eta) & X_{\eta i}(\xi, \eta) & X_{i}(\xi, \eta) / a \\
Y_{\xi i}(\xi, \eta) & Y_{\eta i}(\xi, \eta) & Y_{i}(\xi, \eta) / a \\
Z_{\xi i}(\xi, \eta) & Z_{\eta i}(\xi, \eta) & Z_{i}(\xi, \eta) / a
\end{array}\right]
$$

The derivative of the physical field $q$ in respect to the global coordinate system is then expressed by

$$
\begin{aligned}
& \frac{\partial Q}{\partial x}=P_{x i}(Q: \xi, \eta)=\frac{1}{\left|J_{i}(\xi, \eta)\right|}\left(\frac{\partial \mathscr{P}_{11}(Q: \xi, \eta)}{\partial \xi}+\frac{\partial \mathscr{P}_{12}(Q: \xi, \eta)}{\partial \eta}\right), \\
& \frac{\partial Q}{\partial y}=P_{y i}(Q: \xi, \eta)=\frac{1}{\left|J_{i}(\xi, \eta)\right|}\left(\frac{\partial \mathscr{P}_{21}(Q: \xi, \eta)}{\partial \xi}+\frac{\partial \mathscr{P}_{22}(Q: \xi, \eta)}{\partial \eta}\right), \\
& \frac{\partial Q}{\partial z}=P_{z i}(Q: \xi, \eta)=\frac{1}{\left|J_{i}(\xi, \eta)\right|}\left(\frac{\partial \mathscr{P}_{31}(Q: \xi, \eta)}{\partial \xi}+\frac{\partial \mathscr{P}_{32}(Q: \xi, \eta)}{\partial \eta}\right),
\end{aligned}
$$

where

$$
\left[\begin{array}{ll}
\mathscr{P}_{11}(Q: \xi, \eta) & \mathscr{P}_{12}(Q: \xi, \eta) \\
\mathscr{P}_{21}(Q: \xi, \eta) & \mathscr{P}_{22}(Q: \xi, \eta) \\
\mathscr{P}_{31}(Q: \xi, \eta) & \mathscr{P}_{32}(Q: \xi, \eta)
\end{array}\right]=\frac{P_{i}(Q: \xi, \eta)}{a}\left[\begin{array}{ll}
Y_{\eta i}(\xi, \eta) Z_{i}(\xi, \eta)-Z_{\eta i}(\xi, \eta) Y_{i}(\xi, \eta) & Z_{\eta i}(\xi, \eta) Y_{i}(\xi, \eta)-Y_{\eta i}(\xi, \eta) Z_{i}(\xi, \eta) \\
Z_{\eta i}(\xi, \eta) X_{i}(\xi, \eta)-X_{\eta i}(\xi, \eta) Z_{i}(\xi, \eta) & X_{\eta i}(\xi, \eta) Z_{i}(\xi, \eta)-Z_{\eta i}(\xi, \eta) X_{i}(\xi, \eta) \\
X_{\eta i}(\xi, \eta) Y_{i}(\xi, \eta)-Y_{\eta i}(\xi, \eta) X_{i}(\xi, \eta) & Y_{\eta i}(\xi, \eta) X_{i}(\xi, \eta)-X_{\eta i}(\xi, \eta) Y_{i}(\xi, \eta)
\end{array}\right]
$$

\subsection{Velocity restriction along the surface of the sphere}

In order to guarantee that the direction of the velocity or momentum vector is locally restricted in the tangential directions of the sphere surface, we make use of the projection matrix $\mathscr{A}$ introduced in [58],

$$
\mathscr{A}=\frac{1}{a^{2}}\left[\begin{array}{ccc}
a^{2}-x^{2} & -x y & -x z \\
-x y & a^{2}-y^{2} & -y z \\
-x z & -y z & a^{2}-z^{2}
\end{array}\right]
$$

The momentum vector $\mathbf{m}=(h u, h v, h w)$ needs to be corrected by $\mathscr{A} \cdot \mathbf{m}$. Because the unknowns in the MCV method are the point values, the projection computation is point-wise and hence easy without losing accuracy.

\section{Multi-moment constrained formulation on the sphere}

The surface of the sphere has been partitioned into non-overlapping triangular elements or cells $s_{i}\left(i=1, \ldots, N_{e}\right)$, and $N_{e}=20 N^{2}$ is the total number of triangular elements, where $N$ is the number by which each edge of the icosahedral triangle element is divided as shown in Appendix A. Moreover, we denote the position vector of the point where the value of the physical field is defined by $\mathbf{p}_{i_{p}}=\left(x_{i_{p}}, y_{i_{p}}, z_{i_{p}}\right)$ with $i_{p}=1, \ldots, N_{p}$, and $N_{p}$ is the total number of DOFs over cell $s_{i}$.

Two kinds of discretized quantities, i.e. the cell-averaged value and point value, are then defined as follows:

$$
\begin{aligned}
& \bar{Q}_{i}(t) \equiv \frac{1}{\Delta s_{i}} \int_{s_{i}} Q(x, y, z, t) d s, \\
& Q_{i_{p}}(t) \equiv Q\left(x_{i_{p}}, y_{i_{p}}, z_{i_{p}}, t\right),
\end{aligned}
$$

where $\Delta s_{i}$ is the area of cell $s_{i}$.

As in the context of the multi-moment method, we call $\bar{Q}$ the volume-integrated average (VIA) moment and $Q_{i_{p}}$ the point value (PV) moment. In the multi-moment constrained finite volume (MCV) method, we only use the point values of the prognostic variables defined at $\mathbf{p}_{i_{p}}$ as the unknowns for triangular cell $s_{i}$. The evolution equations for updating the unknowns are derived from the constraints for the moments defined by (3.1).

\subsection{Semi-discrete form}

Eq. (2.1) without the source term is discretized by the finite volume formulation over triangular cell $s_{i}$ as,

$$
\frac{d \bar{Q}_{i}}{d t}=-\frac{1}{\Delta s_{i}} \oint_{l}\left(F n_{x}+G n_{y}+H n_{z}\right) d l=-\frac{1}{\Delta s_{i}} \sum_{j=1}^{3} l_{i j}\left(\bar{F}_{i j} n_{x i j}+\bar{G}_{i j} n_{y i j}+\bar{H}_{i j} n_{z i j}\right),
$$


where $\bar{F}_{i j}, \bar{G}_{i j}, \bar{H}_{i j}$ are the averaged numerical fluxes. Let $\tilde{s}_{i}$ be the corresponding element of $s_{i}$ in the local coordinate $(\xi, \eta)$, the projection of the area $\Delta s_{i}$ of the triangular cell $s_{i}$ onto the local coordinate is

$$
\Delta s_{i}=\int_{s_{i}} d s=\int_{\tilde{s}_{i}}\left|J_{i}(\xi, \eta)\right| d \tilde{s}=2 \Delta\left|J_{i}\right|,
$$

with $\Delta$ being the unit area in the local coordinate. Assuming the three vertices of triangular cell $s_{i}$ to be $\mathbf{p}_{i 1}$, $\mathbf{p}_{i 2}$ and $\mathbf{p}_{i 3}$, we denote the cell edges by $l_{i 1}=\overline{\mathbf{p}_{i 1} \mathbf{p}_{i 2}}, l_{i 2}=\overline{\mathbf{p}_{i 2} \mathbf{p}_{i 3}}$ and $l_{i 3}=\overline{\mathbf{p}_{i 3} \mathbf{p}_{i 1}}$. The outward unit normal vector, $\mathbf{n}_{i j}=\left(n_{x i j}, n_{y i j}, n_{z i j}\right) ;$ with $j=1,2,3$, for each edge is then evaluated by

$$
\mathbf{n}_{i 1}=-\frac{\mathbf{p}_{i 1} \times \mathbf{p}_{i 2}}{\left|\mathbf{p}_{i 1} \| \mathbf{p}_{i 2}\right|}, \quad \mathbf{n}_{i 2}=-\frac{\mathbf{p}_{i 2} \times \mathbf{p}_{i 3}}{\left|\mathbf{p}_{i 2}\right|\left|\mathbf{p}_{i 3}\right|} \quad \text { and } \quad \mathbf{n}_{i 3}=-\frac{\mathbf{p}_{i 3} \times \mathbf{p}_{i 1}}{\left|\mathbf{p}_{i 3}\right|\left|\mathbf{p}_{i 1}\right|} .
$$

The cell-averaged value in (3.2) is evaluated in the local coordinate system,

$$
\bar{Q}_{i}=\frac{\int_{s_{i}} Q_{i}(x, y, z) d s}{\int_{s_{i}} d s}=\frac{\int_{\tilde{s}_{i}}\left|J_{i}(\xi, \eta)\right| Q_{i}(\xi, \eta) d \tilde{s}}{\int_{\tilde{s}_{i}}\left|J_{i}(\xi, \eta)\right| d \tilde{s}},
$$

and the integration of the flux on the cell boundary in (3.2) can be evaluated in the local coordinate by

$$
\left\{\begin{array}{l}
l_{i j} \bar{F}_{i j}=\int_{\tilde{l}_{i j}} \widetilde{J}(\tilde{\xi}) \mid F(\tilde{\xi}) d \tilde{l}, \\
l_{i j} \bar{G}_{i j}=\int_{\tilde{l}_{i j}} \widetilde{J}(\tilde{\xi}) \mid G(\tilde{\xi}) d \tilde{l}, \\
l_{i j} \bar{H}_{i j}=\int_{\tilde{l}_{i j}} \widetilde{J}(\tilde{\xi}) \mid H(\tilde{\xi}) d \tilde{l},
\end{array}\right.
$$

where $\tilde{l}$ represents the edge in the local coordinate system $\tilde{\xi}$, and

$$
\widetilde{J}(\tilde{\xi})|=| \mathbf{p}_{\tilde{\xi}}(\tilde{\xi}) \mid
$$

with $\mathbf{p}$ being the position vector.

Meanwhile, the shallow water equations (2.1) without source term can be also written at point $\mathbf{p}_{i_{p}}$ point-wisely as,

$$
\frac{d Q_{i_{p}}}{d t}=-\left(\widehat{F}_{x i_{p}}+\widehat{G}_{y i_{p}}+\widehat{H}_{z i_{p}}\right)
$$

where $\widehat{F}_{x i_{p}}, \widehat{G}_{y i_{p}}$ and $\widehat{H}_{z i_{p}}$ are the numerical fluxes approximated by solving the Riemann problem in terms of the derivatives.

In the MCV method, we construct a set of time evolution equations for both VIA and PV moments, such as (3.2) and (3.8). The evolution equations for updating the unknowns are then derived from (3.2) and (3.8) which work as the constrained conditions.

Next, we present the third- and fourth order MCV methods as two practical formulations.

\subsubsection{Third order MCV method}

In the third order MCV method, we use the $\mathrm{P}^{+}$triangular element in order to make use of the constrained conditions for both VIA and PV moments. For each triangular element, the unknowns are the PV moments defined as $Q_{i l}(l=1, \ldots, 7)$, respectively, at the cell vertices, the center points of boundary edges and the cell center. As shown in Fig. 3(a), the locations of the seven unknowns in the local coordinate system are

$$
\left\{\begin{array}{l}
\mathbf{a}_{i 1} \equiv(0,0) \\
\mathbf{a}_{i 2} \equiv(1,0) \\
\mathbf{a}_{i 3} \equiv(0,1) \\
\mathbf{a}_{i 4} \equiv\left(\frac{1}{2}, 0\right) \\
\mathbf{a}_{i 5} \equiv\left(\frac{1}{2}, \frac{1}{2}\right) \\
\mathbf{a}_{i 6} \equiv\left(0, \frac{1}{2}\right) \\
\mathbf{a}_{i 7} \equiv\left(\frac{1}{3}, \frac{1}{3}\right)
\end{array}\right.
$$

Then, a set of basis functions for a bi-quadratic Lagrange polynomial in (2.7) and (2.8) are given by

$$
\left\{\begin{array}{l}
c_{i 1}(\xi, \eta)=-3 \xi^{2} \eta-3 \xi \eta^{2}+2 \xi^{2}+2 \eta^{2}+7 \xi \eta-3 \xi-3 \eta+1, \\
c_{i 2}(\xi, \eta)=-3 \xi^{2} \eta-3 \xi \eta^{2}+2 \xi^{2}+3 \xi \eta-\xi \\
c_{i 3}(\xi, \eta)=-3 \xi^{2} \eta-3 \xi \eta^{2}+2 \eta^{2}+3 \xi \eta-\eta \\
c_{i 4}(\xi, \eta)=12 \xi^{2} \eta+12 \xi \eta^{2}-4 \xi^{2}-16 \xi \eta+4 \xi \\
c_{i 5}(\xi, \eta)=12 \xi^{2} \eta+12 \xi \eta^{2}-8 \xi \eta \\
c_{i 6}(\xi, \eta)=12 \xi^{2} \eta+12 \xi \eta^{2}-4 \eta^{2}-16 \xi \eta+4 \eta, \\
c_{i 7}(\xi, \eta)=-27 \xi^{2} \eta-27 \xi \eta^{2}+27 \xi \eta
\end{array}\right.
$$




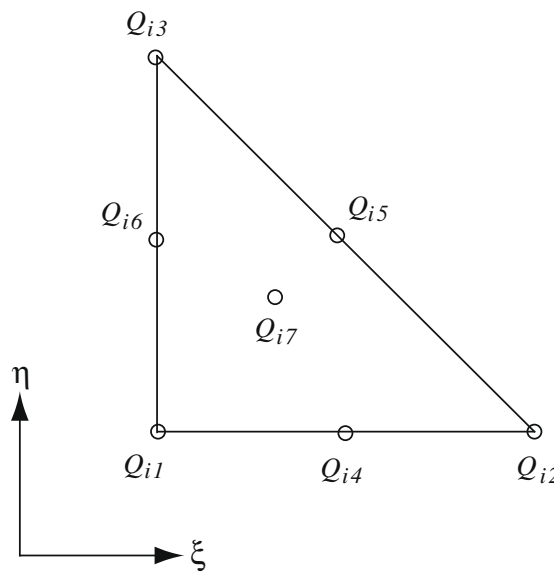

(a) 3rd order MCV

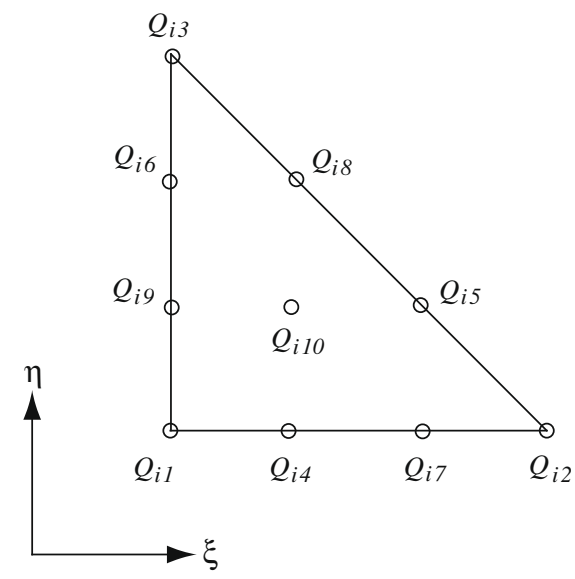

(b) 4th order MCV

Fig. 3. Locations of the unknowns (PV moments).

The PV moments at the cell vertices and the center points of boundary edges, i.e. $Q_{i l}(l=1, \ldots, 6)$, are treated as the unknowns and updated by

$$
\frac{d Q_{i l}}{d t}=-\left(\widehat{F}_{x i l}+\widehat{G}_{y i l}+\widehat{H}_{z i l}\right)=-\mathscr{F}_{i l} \quad(l=1, \ldots, 6),
$$

where we denote $\mathscr{F}_{i l}=\widehat{F}_{x i l}+\widehat{G}_{y i l}+\widehat{H}_{z i l}$ for simplicity. The derivatives of the flux functions $\widehat{F}_{x i l}$, $\widehat{G}_{\text {yil }}$ and $\widehat{H}_{z i l}$ are computed by solving the derivative Riemann problem as will be shown later.

On the other hand, the VIA moment is updated by the flux-form,

$$
\frac{d \bar{Q}_{i}}{d t}=-\frac{1}{\Delta s_{i}} \sum_{j=1}^{3} l_{i j}\left(\widehat{F}_{i j} n_{x i j}+\widehat{G}_{i j} n_{y i j}+\widehat{H}_{i j} n_{z i j}\right)=-\overline{\mathscr{F}}_{i} .
$$

The VIA moment $\bar{Q}_{i}$ can be easily evaluated by the Lagrange polynomial (2.8) with the basis functions (3.10) in terms of seven PV moments $Q_{i l}(l=1, \ldots, 7)$ as,

$$
\begin{aligned}
\bar{Q}_{i} & =\frac{\int_{s_{i}} P_{i}(Q: x, y) d s}{\int_{s_{i}} d s}=\frac{\int_{\tilde{s}_{i}} J_{i}(\xi, \eta) \mid P_{i}(Q: \xi, \eta) d \tilde{s}}{\int_{\tilde{s}_{i}}\left|J_{i}(\xi, \eta)\right| d \tilde{s}} \\
& =\left(\frac{1}{20}\left(\left|J_{i 1}\right| Q_{i 1}+\left|J_{i 2}\right| Q_{i 2}+\left|J_{i 3}\right| Q_{i 3}\right)+\frac{2}{15}\left(\left|J_{i 4}\right| Q_{i 4}+\left|J_{i 5}\right| Q_{i 5}+\left|J_{i 6}\right| Q_{i 6}\right)+\frac{9}{20}\left|J_{i 7}\right| Q_{i 7}\right) / \Delta\left|J_{i}\right| .
\end{aligned}
$$

With (3.11)-(3.13), we can obtain the following time evolution equation for another unknown, the PV at the cell center $Q_{i 7}$,

$$
\frac{d Q_{i 7}}{d t}=\left(\frac{1}{9}\left(\left|J_{i 1}\right| \mathscr{F}_{i 1}+\left|J_{i 2}\right| \mathscr{F}_{i 2}+\left|J_{i 3}\right| \mathscr{F}_{i 3}\right)+\frac{8}{27}\left(\left|J_{i 4}\right| \mathscr{F}_{i 4}+J_{i 5}\left|\mathscr{F}_{i 5}+\right| J_{i 6} \mid \mathscr{F}_{i 6}\right)-\frac{20}{9} \Delta\left|J_{i}\right| \overline{\mathscr{F}}_{i}\right) /\left|J_{i 7}\right| \text {. }
$$

The numerical fluxes $\widehat{\mathbf{F}}_{i l}=\left(\widehat{F}_{i l}, \widehat{G}_{i l}, \widehat{H}_{i l}\right)$ located at $\mathbf{a}_{i l}=\left(\xi_{i l}, \eta_{i l}\right), l=1, \ldots, 6$, are evaluated directly from the PVs readily obtained on the boundary edges of cell $s_{i}$.

The integration of the flux functions $\overline{\mathbf{F}}_{i j}=\left(\bar{F}_{i j}, \bar{G}_{i j}, \bar{H}_{i j}\right)$ along each cell-boundary edge $l_{i j}(j=1,2,3)$ as shown in (3.6) can be approximately computed by

$$
\left\{\begin{array}{l}
l_{i 1} \overline{\mathbf{F}}_{i 1}=\frac{\widetilde{J}_{i 1} \widehat{\mathbf{F}}_{i 1}+\widetilde{J}_{i 2} \mid \widehat{\mathbf{F}}_{i 2}+4 \widetilde{J}_{i 4} \widehat{\mathbf{F}}_{i 4}}{6}, \\
l_{i 2} \overline{\mathbf{F}}_{i 2}=\frac{\widetilde{J}_{i 2} \widehat{\mathbf{F}}_{i 2}+\widetilde{J}_{i 3} \mid \widehat{\mathbf{F}}_{i 3}+4 \widetilde{J}_{i 5} \widehat{\mathbf{F}}_{i 5}}{6}, \\
l_{i 3} \overline{\mathbf{F}}_{i 3}=\frac{\widetilde{J}_{i 3} \widehat{\mathbf{F}}_{i 3}+\widetilde{J}_{i 1} \mid \widehat{\mathbf{F}}_{i 1}+4 \widetilde{J}_{i 6} \widehat{\mathbf{F}}_{i 6}}{6},
\end{array}\right.
$$

It is observed that formula (3.15) maintains the third order accuracy of the numerical solution. 
3.1.2. Fourth order MCV method

In the fourth order MCV method, we locally define the PV moments as Fig. 3(b). The locations of the points in the local coordinate system are given as,

$$
\left\{\begin{array}{l}
\mathbf{a}_{i 1} \equiv(0,0), \\
\mathbf{a}_{i 2} \equiv(1,0), \\
\mathbf{a}_{i 3} \equiv(0,1), \\
\mathbf{a}_{i 4} \equiv\left(\frac{1}{3}, 0\right), \\
\mathbf{a}_{i 5} \equiv\left(\frac{2}{3}, \frac{1}{3}\right), \\
\mathbf{a}_{i 6} \equiv\left(0, \frac{2}{3}\right), \\
\mathbf{a}_{i 7} \equiv\left(\frac{2}{3}, 0\right), \\
\mathbf{a}_{i 8} \equiv\left(\frac{1}{3}, \frac{2}{3}\right), \\
\mathbf{a}_{i 9} \equiv\left(0, \frac{1}{3}\right), \\
\mathbf{a}_{i 10} \equiv\left(\frac{1}{3}, \frac{1}{3}\right) .
\end{array}\right.
$$

In a similar manner, a set of the basis functions for a cubic polynomial in the local coordinate system can be given by

$$
\left\{\begin{array}{l}
c_{i 1}(\xi, \eta)=-\frac{9}{2} \xi^{3}-\frac{9}{2} \eta^{3}-\frac{27}{2} \xi^{2} \eta-\frac{27}{2} \xi \eta^{2}+9 \xi^{2}+9 \eta^{2}+18 \xi \eta-\frac{11}{2} \xi-\frac{11}{2} \eta+1, \\
c_{i 2}(\xi, \eta)=\frac{9}{2} \xi^{3}-\frac{9}{2} \xi^{2}+\xi \\
c_{i 3}(\xi, \eta)=\frac{9}{2} \eta^{3}-\frac{9}{2} \eta^{2}+\eta \\
c_{i 4}(\xi, \eta)=\frac{27}{2} \xi^{3}+27 \xi^{2} \eta+\frac{27}{2} \xi \eta^{2}-\frac{45}{2} \xi^{2}-\frac{45}{2} \xi \eta+9 \xi \\
c_{i 5}(\xi, \eta)=\frac{27}{2} \xi^{2} \eta-\frac{9}{2} \xi \eta \\
c_{i 6}(\xi, \eta)=-\frac{27}{2} \eta^{3}-\frac{27}{2} \xi \eta^{2}+18 \eta^{2}+\frac{9}{2} \xi \eta-\frac{9}{2} \eta \\
c_{i 7}(\xi, \eta)=-\frac{27}{2} \xi^{3}-\frac{27}{2} \xi^{2} \eta+18 \xi^{2}+\frac{9}{2} \xi \eta-\frac{9}{2} \xi \\
c_{i 8}(\xi, \eta)=\frac{27}{2} \xi \eta^{2}-\frac{9}{2} \xi \eta \\
c_{i 9}(\xi, \eta)=\frac{27}{2} \eta^{3}+\frac{27}{2} \xi^{2} \eta+27 \xi \eta^{2}-\frac{45}{2} \eta^{2}-\frac{45}{2} \xi \eta+9 \eta \\
c_{i 10}(\xi, \eta)=-27 \xi^{2} \eta-27 \xi \eta^{2}+27 \xi \eta
\end{array}\right.
$$

Analogously, the PV moments at cell vertices and cell edges are updated by

$$
\frac{d Q_{i l}}{d t}=-\left(\widehat{F}_{x i l}+\widehat{G}_{y i l}+\widehat{H}_{z i l}\right)=-\mathscr{F}_{i l} \quad(l=1, \ldots, 9),
$$

and the VIA moment is updated by

$$
\frac{d \bar{Q}_{i}}{d t}=-\frac{1}{\Delta s_{i}} \sum_{j=1}^{3} l_{i j}\left(\bar{F}_{i j} n_{x i j}+\bar{G}_{i j} n_{y i j}+\bar{H}_{i j} n_{z i j}\right)=-\overline{\mathscr{F}}_{i} .
$$

From the cubic polynomial basis function (2.8) and (3.17), the VIA moment can be expressed by,

$$
\bar{Q}_{i}=\left(\frac{1}{30}\left(\left|J_{i 1}\right| Q_{i 1}+\left|J_{i 2}\right| Q_{i 2}+\left|J_{i 3}\right| Q_{i 3}\right)+\frac{3}{40}\left(\left|J_{i 4}\right| Q_{i 4}+\left|J_{i 5}\right| Q_{i 5}+\left|J_{i 6}\right| Q_{i 6}+\left|J_{i 7}\right| Q_{i 7}+\left|J_{i 8}\right| Q_{i 8}+\left|J_{i 9}\right| Q_{i 9}\right)+\frac{9}{20}\left|J_{i 10}\right| Q_{i 10}\right) / \Delta\left|J_{i}\right| .
$$

Finally, we arrive at the following time evolution equation for $Q_{i 10}$,

$$
\frac{d Q_{i 10}}{d t}=\left(\frac{2}{27}\left(\left|J_{i 1}\right| \mathscr{F}_{i 1}+J_{i 2}\left|\mathscr{F}_{i 2}+\right| J_{i 3} \mid \mathscr{F}_{i 3}\right)+\frac{1}{6}\left(\left|J_{i 4}\right| \mathscr{F}_{i 4}+\left|J_{i 5}\right| \mathscr{F}_{i 5}+\left|J_{i 6}\right| \mathscr{F}_{i 6}+\left|J_{i 7}\right| \mathscr{F}_{i 7}+\left|J_{i 8}\right| \mathscr{F}_{i 8}+\left|J_{i 9}\right| \mathscr{F}_{i 9}\right)-\frac{20}{9} \Delta\left|J_{i}\right| \overline{\mathscr{F}}_{i}\right) /\left|J_{i 10}\right| .
$$

The integration of the flux functions $\overline{\mathbf{F}}_{i j}=\left(\bar{F}_{i j}, \bar{G}_{i j}, \bar{H}_{i j}\right)$ along cell-boundary edge $l_{i j}(j=1,2,3)$ as shown in (3.6) can be computed with a fourth order accuracy by

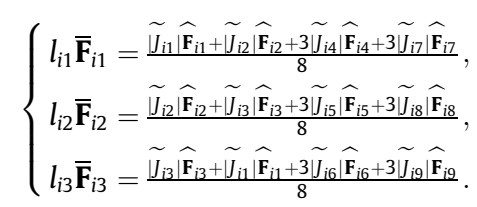




\subsection{Approximate Riemann solver}

We apply the generalized Riemann problem suggested in [53,52] and used in [34,35] to evaluate the numerical fluxes in term of the derivative at point $\mathbf{p}_{i_{p}}=\left(x_{i_{p}}, y_{i_{p}}, z_{i_{p}}\right)$ on the cell boundary edges.

Given the unknowns $Q_{i l}$, we obtain the piecewise reconstruction over each single cell by Lagrange polynomial (2.8) for the flux functions $F(Q), G(Q)$ and $H(Q)$, respectively. We denote the reconstruction interpolation functions for the cells adjacent to $\mathbf{p}_{i_{p}}$ from $x_{i_{p}}^{+}$side and $x_{i_{p}}^{-}$side by $P^{x+}\left(F(Q): \xi_{i_{p}}, \eta_{i_{p}}\right)$ and $P^{x-}\left(F(Q): \xi_{i_{p}}, \eta_{i_{p}}\right)$, respectively. In the same manner, we have the two adjacent reconstruction interpolations in $y$ direction as $P^{y+}\left(G(Q): \xi_{i_{p}}, \eta_{i_{p}}\right)$ and $P^{y-}\left(G(Q): \xi_{i_{p}}, \eta_{i_{p}}\right)$, and in $z$ direction as $P^{z+}\left(H(Q): \xi_{i_{p}}, \eta_{i_{p}}\right)$ and $P^{z-}\left(H(Q): \xi_{i_{p}}, \eta_{i_{p}}\right)$

As mentioned before, the unknowns $Q_{i_{p}}$ defined at the cell boundary are continuous and shared by the neighboring cells. Thus, the flux functions are computed directly from $Q_{i_{p}}$ by

$$
\left\{\begin{array}{l}
\widehat{F}_{i_{p}}=F\left(Q_{i_{p}}\right), \\
\widehat{G}_{i_{p}}=G\left(Q_{i_{p}}\right), \\
\widehat{H}_{i_{p}}=H\left(Q_{i_{p}}\right) .
\end{array}\right.
$$

The derivatives of the flux functions, however, might be discontinuous at $\mathbf{p}_{i_{p}}$. Therefore, we evaluate the derivatives of flux functions from the two different states from the adjacent reconstruction interpolation functions by approximate Riemann solver, namely $\mathscr{R}$ iemann $(\cdot, \cdot)$. The numerical derivatives of the flux functions is then written in form as,

$$
\left\{\begin{array}{l}
\widehat{F}_{x i_{p}}=\mathscr{R i e m a n n}\left(F_{x}^{x-}, F_{x}^{x+}\right), \\
\widehat{G}_{y i_{p}}=\mathscr{R i e m a n n}\left(G_{y}^{y-}, G_{y}^{y+}\right), \\
\widehat{H}_{z i_{p}}=\mathscr{R i e m a n n}\left(H_{z}^{z-}, H_{z}^{z+}\right),
\end{array}\right.
$$

where the derivatives in respect to $x, y$ and $z$ for all neighboring cells are computed from the cell-wise interpolation reconstructions by

$$
\begin{aligned}
& \left\{\begin{array}{l}
F_{x i_{p}}^{x-}=\frac{\partial}{\partial x} P^{x-}\left(F(Q): \xi_{i_{p}}, \eta_{i_{p}}\right), \\
F_{x i_{p}}^{x+}=\frac{\partial}{\partial x} P^{x+}\left(F(Q): \xi_{i_{p}}, \eta_{i_{p}}\right),
\end{array}\right. \\
& \left\{\begin{array}{l}
G_{y i_{p}}^{y-}=\frac{\partial}{\partial y} P^{y-}\left(G(Q): \xi_{i_{p}}, \eta_{i_{p}}\right), \\
G_{y i_{p}}^{y+}=\frac{\partial}{\partial y} P^{y+}\left(G(Q): \xi_{i_{p}}, \eta_{i_{p}}\right),
\end{array}\right. \\
& \left\{\begin{array}{l}
H_{z i_{p}}^{z-}=\frac{\partial}{\partial z} P^{z-}\left(H(Q): \xi_{i_{p}}, \eta_{i_{p}}\right), \\
H_{z i_{p}}^{z+}=\frac{\partial}{\partial z} P^{z+}\left(H(Q): \xi_{i_{p}}, \eta_{i_{p}}\right) .
\end{array}\right.
\end{aligned}
$$

In this paper, we apply a flux vector splitting to evaluate the approximate Riemann solver. The approximate flux derivative reads, for example, in $x$ direction as,

$$
\widehat{F}_{x i_{p}}=\mathscr{R i e m a n n}\left(F_{x}^{x-}, F_{x}^{x+}\right)=\frac{1}{2}\left(F_{x i_{p}}^{x-}+F_{x i_{p}}^{x+}-R_{i_{p}} \operatorname{sign}\left(\Lambda_{i_{p}}\right) R_{i_{p}}^{-1}\left(F_{x i_{p}}^{x+}-F_{x i_{p}}^{x-}\right)\right),
$$

where $\Lambda$ is the eigen value diagonal matrix of $\partial F / \partial Q$, and $R$ and $R^{-1}$ the corresponding matrix of right and left eigenvectors (see [40] for detail), which all are directly evaluated by the unknowns at $\mathbf{p}_{i_{p}}$. The same formulation applies to $y$ and $z$ directions.

When including the source terms of the topographic effect, we cast the geopotential gradient force in terms of the total height of the wave surface to keep the balance between the inviscid flux and the bottom topographic effect. The source terms of topography in (3.4), i.e. $g b T_{x}, g b T_{y}$ and $g b T_{z}$, needed to be computed in a way consistent with flux splitting (3.26).

Noting that the bottom topography $b$ is continuous at the cell boundary, but the derivatives of the total height $T_{x}, T_{y}$ and $T_{z}$ might be discontinuous, we adopt a formula similar to (3.26) to evaluate the source term for the $x$ component as,

$$
\left(g b \widehat{T}_{x}\right)_{i_{p}}=\frac{1}{2} g b_{i_{p}}\left(T_{x i_{p}}^{-}+T_{x i_{p}}^{+}-R_{i_{p}} \operatorname{sign}\left(\Lambda_{i_{p}}\right) R_{i_{p}}^{-1}\left(T_{x i_{p}}^{+}-T_{x i_{p}}^{-}\right)\right) .
$$

The above applies to $g b T_{y}$ and $g b T_{z}$ in $y$ and $z$ directions, respectively.

The above treatment guarantees exactly the balance between the numerical flux and the topographic source term to satisfy the C-property, thus eliminate the topography-induced spurious oscillation which may be generated in the mountain wave test in [58] for example.

\subsection{Time integration}

We apply the third order TVD Runge-Kutta time integration method [46] to the semi-discretized equations of the unknowns, i.e. (3.11) and (3.14) for the third order scheme or (3.18) and (3.21) for the fourth order scheme. We write the semi-discretized equations in the form as, 


$$
\frac{d Q}{d t}=R(Q),
$$

where $R(Q)$ stands for the spatial approximations for the flux functions and their derivatives discussed above.

Given the values $Q^{n}$ at step $n$, the third order TVD Runge-Kutta method yields the following multi-step updating to obtain the values $Q^{n+1}$ at step $n+1$,

$$
\begin{aligned}
& Q_{0}=Q^{n}, \\
& Q_{1}=Q_{0}+\Delta t R\left(Q_{0}\right), \\
& Q_{2}=\frac{3}{4} Q_{0}+\frac{1}{4} Q_{1}+\frac{1}{4} \Delta t R\left(Q_{1}\right), \\
& Q_{3}=\frac{1}{3} Q_{0}+\frac{2}{3} Q_{2}+\frac{2}{3} \Delta t R\left(Q_{2}\right), \\
& Q^{n+1}=Q_{3} .
\end{aligned}
$$

As discussed before, one needs to project the momentum vector with matrix $\mathscr{A}$ given in (2.12) to remain the motion on the surface of the sphere. We denote the projection operation by

$$
\mathscr{P}_{\text {roject }}(Q)=\widetilde{A} Q
$$

with

$$
\widetilde{A}=\frac{1}{a^{2}}\left[\begin{array}{cccc}
a^{2} & 0 & 0 & 0 \\
0 & a^{2}-x^{2} & -x y & -x z \\
0 & -x y & a^{2}-y^{2} & -y z \\
0 & -x z & -y z & a^{2}-z^{2}
\end{array}\right] .
$$

Thus, updating procedure (3.29) is modified to

$$
\begin{aligned}
& Q_{0}=Q^{n}, \\
& Q_{1}=\mathscr{P}_{\text {roject }}\left(Q_{0}+\Delta t R\left(Q_{0}\right)\right), \\
& Q_{2}=\mathscr{P}_{\text {roject }}\left(\frac{3}{4} Q_{0}+\frac{1}{4} Q_{1}+\frac{1}{4} \Delta t R\left(Q_{1}\right)\right), \\
& Q_{3}=\mathscr{P}_{\text {roject }}\left(\frac{1}{3} Q_{0}+\frac{2}{3} Q_{2}+\frac{2}{3} \Delta t R\left(Q_{2}\right)\right), \\
& Q^{n+1}=Q_{3} .
\end{aligned}
$$

It is noted that all predicted unknowns in the MCV method are point values, so the point-wise projection can be computed accurately.

\subsection{Remarks on the computational efficiency in comparison with other existing methods}

In the MCV methods presented in this paper, all unknowns are defined at the cell vertices and boundary edges except the only one PV at the cell center. So, the neighboring cells share the unknowns at the cell vertices and cell edges, which significantly reduces the total number of the unknowns compared to other existing schemes that locate the unknowns (DOFs) inside mesh cells.

Next, we give a quantitative comparison of the total DOFs of the third order MCV scheme with its DG counterpart. Consider that each icosahedral triangle is partitioned into $N^{2}$ grid elements as described at the beginning of Section 3 , the total numbers of vertices $\left(N_{v}\right)$, cell boundary edges $\left(N_{b}\right)$ and elements $\left(N_{e}\right)$ are

$$
\left\{\begin{array}{l}
N_{v}=10 N^{2}+2, \\
N_{b}=30 N^{2}, \\
N_{e}=20 N^{2} .
\end{array}\right.
$$

The total number of PV moments (or DOFs) $N_{p}$ for a physical variable is given as $N_{p}=N_{v}+N_{b}+N_{e}=60 N^{2}+2$, while the DG method with a $P^{2}$ polynomial requires $N_{p}=6 \times N_{e}=120 N^{2}$ DOFs in total. It is obvious that an MCV method on triangular mesh requires less memory comparing to a DG method of the same order accuracy.

We should also note that the flux function along the cell boundary can be directly computed from the PV moment that are updated as computational variable, and the updating of each PV moment on the cell boundary needs point-wise derivative Riemann solver. The total count of this is $N_{r}=N_{v}+N_{b}=40 N^{2}+2$ in the third order scheme, while that for DG method is $N_{r}=2 \times N_{b}=60 N^{2}$.

Moreover, the DG method requires high order numerical integration which is not needed in the MCV formulation. The merit that all computational variables are point values in the MCV method also provides great convenience in accurately calculating metric terms on a curved surface and source terms of physical processes in real applications. In our numerical 
test, we found that for the schemes of same order and same time marching algorithm (Runge-Kutta), MCV method has larger CFL number for computational stability compared to the results of DG and SV (Spectral Volume) methods reported in [69].

From above discussions, we know that the MCV method has obvious superiority on computational efficiency.

\section{Numerical examples}

In this section, we will present several widely used numerical examples for global transport and shallow water models to evaluate the proposed numerical model. The convergence rate of the numerical model is examined by grid refinement tests for the global advection transport problem and the balanced geostrophic flow. The standard benchmark tests for both advection equation and shallow water equations proposed in [58] and other newly suggested tests are used to examine the performance of the numerical model from different aspects.

The relationship between the Cartesian coordinate and the longitude-latitude coordinate is given by

$$
\left\{\begin{array}{l}
x=a \cos \lambda \cos \theta \\
y=a \sin \lambda \cos \theta \\
z=a \sin \theta
\end{array}\right.
$$

where $\lambda$ is the longitude and $\theta$ the latitude.

As in [58], the initial velocity field is given in the longitude-latitude coordinate $(\lambda \in[0,2 \pi], \theta \in[-\pi / 2, \pi / 2])$, and then converted to the 3D Cartesian coordinate as follows,

$$
\begin{aligned}
& \mathbf{u}=\mathscr{B} \mathbf{u}^{(L)}, \\
& \mathscr{B}=\left[\begin{array}{ccc}
-\sin \lambda & -\cos \lambda \sin \theta & 0 \\
\cos \lambda & -\sin \lambda \sin \theta & 0 \\
0 & \cos \theta & 0
\end{array}\right],
\end{aligned}
$$

where $\mathbf{u}=(u, v, w)$ is the velocity vector in the global Cartesian coordinate and $\mathbf{u}^{(L)}=\left(\boldsymbol{u}^{(\lambda)}, \boldsymbol{u}^{(\theta)}, \boldsymbol{u}^{(r)}\right)$ is the velocity defined in the longitude-latitude coordinate by $u^{(\lambda)}=a \cos \theta \dot{\lambda}, u^{(\theta)}=a \dot{\theta}$ and $u^{(r)}=\dot{r}=0$.

We will show numerical results in the $256 \times 128$ longitude-latitude coordinate grid instead of the global coordinate for clarity. The plotted results are interpolated by bi-quadratic or cubic polynomial on a single cell. In this paper, we depict the results in the range of $\lambda \in[-\pi, \pi]$ and $\theta \in[-\pi / 2, \pi / 2]$.

In order to quantitatively examine the numerical outputs, we use the following normalized error measures introduced in [58],

$$
\begin{aligned}
& L_{1}(\psi) \equiv \frac{\int_{\Omega}\left|\psi^{(n)}-\psi^{(e)}\right| d \Omega}{\int_{\Omega}\left|\psi^{(e)}\right| d \Omega}, \\
& L_{2}(\psi) \equiv \frac{\left\{\int_{\Omega}\left(\psi^{(n)}-\psi^{(e)}\right)^{2} d \Omega\right\}^{1 / 2}}{\left\{\int_{\Omega}\left(\psi^{(e)}\right)^{2} d \Omega\right\}^{1 / 2}}, \\
& L_{\infty}(\psi) \equiv \frac{\max \left(\left|\psi^{(n)}-\psi^{(e)}\right|\right)_{\in \Omega}}{\max \left(\left|\psi^{(e)}\right|\right)_{\in \Omega}},
\end{aligned}
$$

and numerical conservation is evaluated by the normalized conservation error for physical variable $\psi$,

$$
M(\psi) \equiv \frac{\int_{\Omega} \psi^{(n)} d \Omega-\int_{\Omega} \psi^{(0)} d \Omega}{\int_{\Omega} \psi^{(0)} d \Omega},
$$

where $\Omega$ is the whole computational domain, and $\psi^{(n)}, \psi^{(e)}$ and $\psi^{(0)}$ are the numerical solution, the exact solution and the initial condition.

The integration operator is computed by

$$
I(\psi) \equiv \int_{\Omega} \psi d \Omega=\sum_{i=1}^{N_{e}} \int_{s_{i}} \psi_{i}(x, y, z) d s=\sum_{i=1}^{N_{e}} \int_{\tilde{s}_{i}}\left|J_{i}(\xi, \eta)\right| \psi_{i}(\xi, \eta) d \tilde{s},
$$

where the numerical quadrature is calculated by (3.13) for the third order scheme or (3.20) for the fourth order scheme.

With (4.5), the errors are numerically calculated by

$$
\begin{aligned}
& L_{1}(\psi)=\frac{I\left(\left|\psi^{(n)}-\psi^{(e)}\right|\right)}{I\left(\left|\psi^{(e)}\right|\right)}, \\
& L_{2}(\psi)=\left\{\frac{I\left(\left(\psi^{(n)}-\psi^{(e)}\right)^{2}\right)}{I\left(\left(\psi^{(e)}\right)^{2}\right)}\right\}^{1 / 2}, \\
& L_{\infty}(\psi)=\frac{\sum_{i_{p}=1}^{N_{p}} \max \left(\left|\psi_{i_{p}}^{(n)}-\psi_{i_{p}}^{(e)}\right|\right)}{\sum_{i_{p}=1}^{N_{p}} \max \left(\left|\psi_{i_{p}}^{(e)}\right|\right)},
\end{aligned}
$$


and

$$
M(\psi)=\frac{I\left(\psi^{(n)}\right)-I\left(\psi^{(0)}\right)}{I\left(\psi^{(0)}\right)} .
$$

\subsection{Global transport model}

First, we consider the advection transport computations for the mass conservation equation of passive tracer $h$,

$$
h_{t}+(h u)_{x}+(h v)_{y}+(h w)_{z}=0 .
$$

\subsubsection{Accuracy study [4]}

We examine the convergence rates of the proposed schemes with the grid refinement tests. The initially smooth profile of $h$ is given as [4],

$$
h(\lambda, \theta, 0)=\sin \lambda \cos \theta .
$$

The velocity fields satisfying the divergence-free condition $\nabla \cdot \mathbf{u}=0$ given in [58] are

$$
\left\{\begin{array}{l}
u^{(\lambda)}=u_{0}(\cos \theta \cos \alpha+\sin \theta \cos \lambda \sin \alpha), \\
u^{(\theta)}=-u_{0} \sin \lambda \sin \alpha,
\end{array}\right.
$$

where $u_{0}=2 \pi a /(12$ days $)$ is the maximum of the velocity, and $\alpha$ is the angle between the rotation axis and the north polar of the Earth.

The computational mesh is doubly refined from $N=2$ to $N=16$. We have run the tests with a rotation angle $\alpha=\pi / 4$ up to 12 days ( 1 period).

The measured errors are shown in Table 1. Both the third and fourth order schemes possess the converging rates as expected. We also calculated other cases with the rotation angle $\alpha$ being 0 and $\pi / 2$ and observed that the error is not sensitive to the flow direction, and hence the schemes are robust to the grid morphology.

\subsubsection{Williamson's test case 1: advection of cosine bell [58]}

The initial cosine bell is given by

$$
h(\lambda, \theta, 0)= \begin{cases}\frac{h_{0}}{2}\left(1+\cos \frac{\pi R}{R}\right), & \text { if } \widehat{R} \leqslant R \\ 0, & \text { if } \widehat{R}>R\end{cases}
$$

where $h_{0}=1000, R=a / 3$ and $\widehat{R}$ is the great circle distance defined as,

$$
\widehat{R}=a \operatorname{acos}\left(\sin \theta_{c} \sin \theta+\cos \theta_{c} \cos \theta \cos \lambda-\lambda_{c}\right)
$$

with the center at $\lambda_{c}=3 \pi / 2$ and $\theta_{c}=0$. The rotational velocity is the same as (4.10). We run the tests with a rotational angle $\alpha=\pi / 4$. We use the mesh of $N=25$ for the third order scheme and the mesh of $N=20$ for the fourth order scheme. As can be seen, the total DOFs for the fourth order MCV method on the $N=20$ mesh is almost the same as that for the third order MCV method on the $N=25$ mesh.

\begin{tabular}{|c|c|c|c|c|c|c|}
\hline$N$ & $L_{1}$ & Order & $L_{2}$ & Order & $L_{\infty}$ & Order \\
\hline \multicolumn{7}{|c|}{ 3rd order $M C V$} \\
\hline 2 & $1.63 \mathrm{E}-1$ & - & $1.84 \mathrm{E}-1$ & - & $1.32 \mathrm{E}-1$ & - \\
\hline 4 & $2.06 \mathrm{E}-2$ & 2.93 & $2.10 \mathrm{E}-2$ & 2.96 & $2.55 \mathrm{E}-2$ & 2.85 \\
\hline 8 & $2.56 \mathrm{E}-3$ & 3.01 & $2.63 E-3$ & 3.00 & $3.05 E-3$ & 3.06 \\
\hline 16 & $3.24 \mathrm{E}-4$ & 2.98 & $3.30 \mathrm{E}-4$ & 2.99 & $3.91 \mathrm{E}-4$ & 2.96 \\
\hline \multicolumn{7}{|c|}{ 4th order $M C V$} \\
\hline 2 & $4.21 \mathrm{E}-2$ & - & $4.20 \mathrm{E}-2$ & - & $5.22 \mathrm{E}-2$ & - \\
\hline 4 & $2.68 \mathrm{E}-3$ & 3.97 & $2.67 \mathrm{E}-3$ & 3.98 & $3.50 \mathrm{E}-3$ & 3.90 \\
\hline 8 & $1.68 \mathrm{E}-4$ & 4.00 & $1.66 \mathrm{E}-4$ & 4.01 & $2.08 \mathrm{E}-4$ & 4.07 \\
\hline 16 & $1.06 \mathrm{E}-5$ & 3.99 & $1.04 \mathrm{E}-5$ & 4.00 & $1.33 \mathrm{E}-5$ & 3.97 \\
\hline
\end{tabular}

We show the numerical results and exact solution after 12 days in Fig. 4. Both the third and fourth order MCV methods produce adequate numerical results without dispersion error. Especially, the fourth order MCV method gives numerical results almost visually identical to the exact solution. We also plot the time history of the normalized errors in Fig. 5. It is ob-

Table 1

Numerical error and convergence rate for the advection of $q_{0}=\cos \lambda \sin \theta$ with the rotational angle $\alpha=\pi / 4$. 


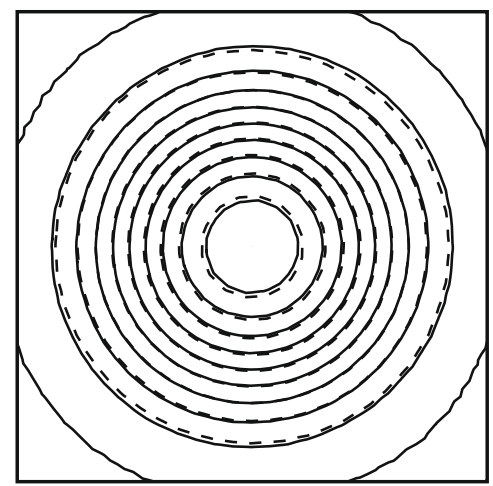

(a) 3rd order $\mathrm{MCV}$ on $N=25$

$(\mathrm{DOFs}=37502)$

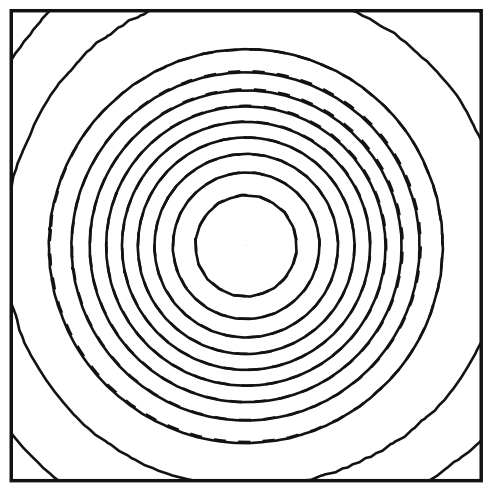

(b) 4th order MCV on $N=20$

$(\mathrm{DOFs}=36002)$

Fig. 4. Numerical solution at day 12 and exact solution for test case 1 with $\alpha=\pi / 4$. The solid line shows numerical result and dashed line shows exact solution. The contour interval is 100 from 0 to 1000 .

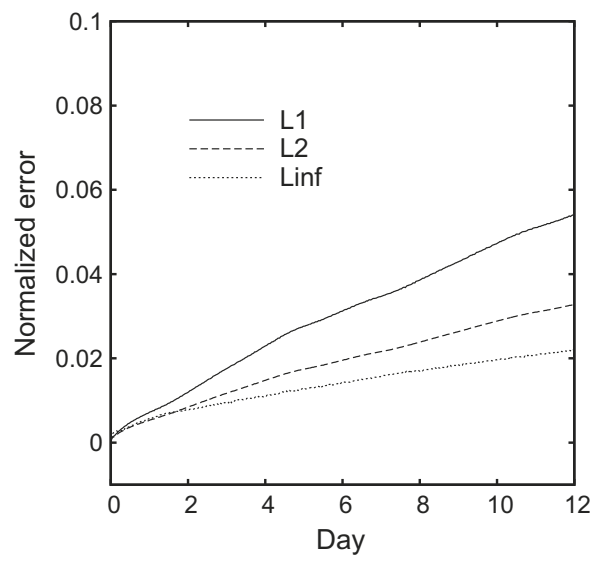

(a) 3rd order MCV on $N=25(\mathrm{DOFs}=37502)$

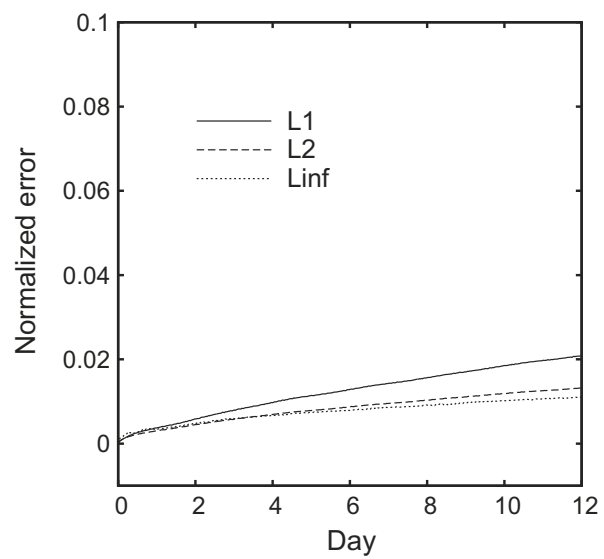

(b) 4th order MCV on $N=20(\mathrm{DOFs}=36002)$

Fig. 5. Normalized errors in test case 1 with $\alpha=\pi / 4$.

served that the result of the fourth order scheme is apparently superior to that of the third order one in numerical errors. It manifests that a higher order scheme is more attractive in accuracy compared to a lower order one with nearly the same number of DOFs.

\subsubsection{Deformational flow on the sphere [30]}

This test was originally proposed on a plane geometry by Doswell [12], and was implemented to the spherical geometry by Nair et al. [29]. The numerical configuration of this test in detail can be found in [30]. In this test, we set the radius of the Earth $a$ to 1 same as [4], and the vortical center is located at the north-pole of the Earth.

The initial condition is given by

$$
h(\lambda, \theta, 0)=1-\tanh \left(\frac{\rho}{\gamma} \sin \lambda\right),
$$

where $\rho=\rho_{0} \cos \theta$ is the radius of the vortex. We specified the parameters as $\rho_{0}=3$ and $\gamma=5$. The velocity field is defined as,

$$
\left\{\begin{array}{l}
u^{(\lambda)}(\lambda, \theta)=\omega \cos \theta \\
u^{(\theta)}(\lambda, \theta)=0
\end{array}\right.
$$

with the angular velocity $\omega$ given by 


$$
\omega= \begin{cases}0, & \text { if } \rho=0, \\ \frac{3 \sqrt{2}}{2 \rho} \operatorname{sech}(\rho) \tanh (\rho), & \text { if } \rho \neq 0 .\end{cases}
$$

Then, the exact solution can be written as,

$$
h^{(e)}(\lambda, \theta, t)=1-\tanh \left(\frac{\rho}{\gamma} \sin (\lambda-\omega t)\right) .
$$

The numerical results at $t=\pi$ with third order method on $N=25$ mesh and fourth order method on $N=20$ mesh are shown in Figs. 6(a) and 7(a). There is no visible difference among the numerical outputs. Thus, we also plotted the difference between the numerical solutions and the exact solution in Figs. 6(b) and 7(b). The numerical errors of different norms of the third order method and fourth order method are $L_{1}=3.2302 \mathrm{E}-5, L_{2}=1.2728 \mathrm{E}-4, L_{\infty}=1.1111 \mathrm{E}-3$, and $L_{1}=7.7886 \mathrm{E}-6, L_{2}=3.2466 \mathrm{E}-5, L_{\infty}=3.8007 \mathrm{E}-4$. Again, with almost same number of DOFs, the result of the fourth order scheme is better than that of the third order scheme. In this test, one center of vortices is set at a vertex of the initial icosahedron which is topologically singular. Nevertheless, numerical results look not sensitive to this.

\subsubsection{Moving vortices on the sphere [32]}

This test is introduced by Nair and Jablonowski in 2008 to evaluate the computation of advection transport with a more challenging deformational velocity field on the sphere. In addition to the deformational flow given in Section 4.1.3, complexity in the velocity field is increased by adding a rotational flow to the vortical motion. The detail of the computational setup follows [32]. We show the numerical results of the third order MCV method on $N=30$ mesh at 3, 6, 9 and 12 (full revolution) days in Fig. 8(a)-(d). The two vortices are initially located on the equator at $90^{\circ} \mathrm{E}$ and $90^{\circ} \mathrm{E}$ and then moved with a rotational angle $\alpha=\pi / 4$. Against Fig. 8(e), which is the analytical solution at 12 days, our result looks adequately comparable to those

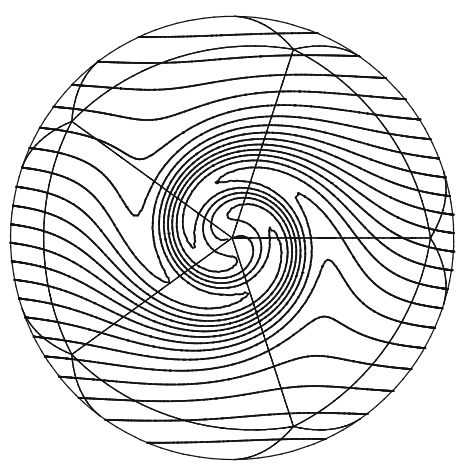

(a) Numerical result

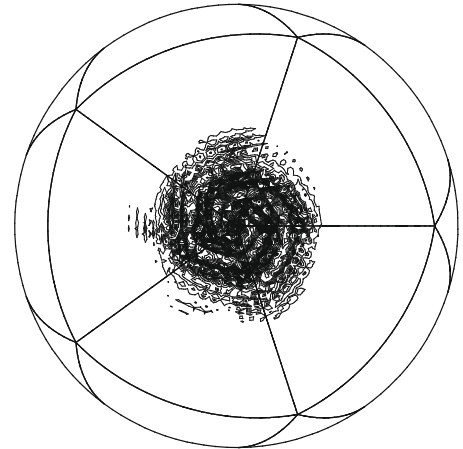

(b) Difference with the exact solution

Fig. 6. Numerical result and difference with the exact solution computed by 3rd order MCV method on $N=25$ (DOFs $=37,502$ ) for the deformational flow at $t=\pi$. The contour interval of the numerical result is 0.05 from 0.5 to 1.5 , and the contour of difference with the exact solution is drawn with the interval 0.0001 from -0.0015 to 0.0015 except 0 .

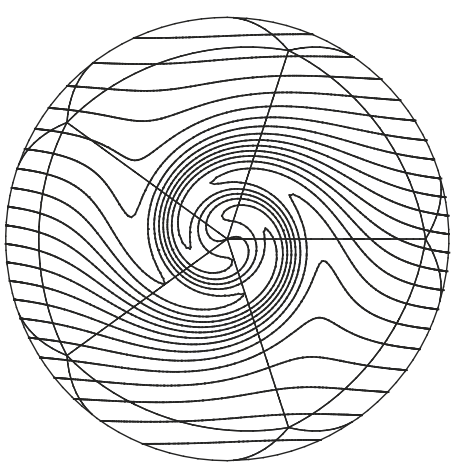

(a) Numerical result

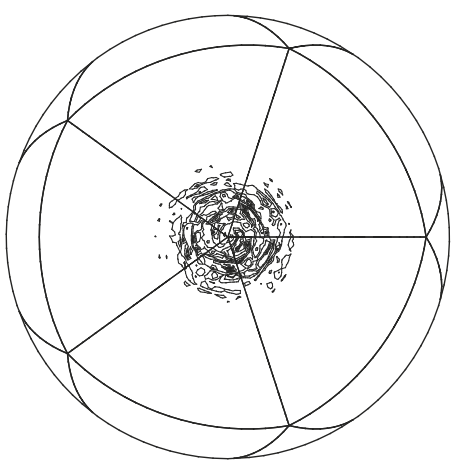

(b) Difference with the exact solution

Fig. 7. Same as Fig. 6, but computed by 4 th order MCV method on $N=20$ (DOFs $=36,002)$. 


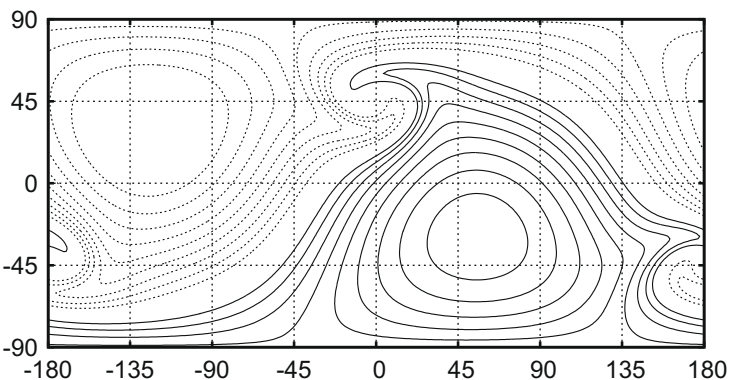

(a) day 3

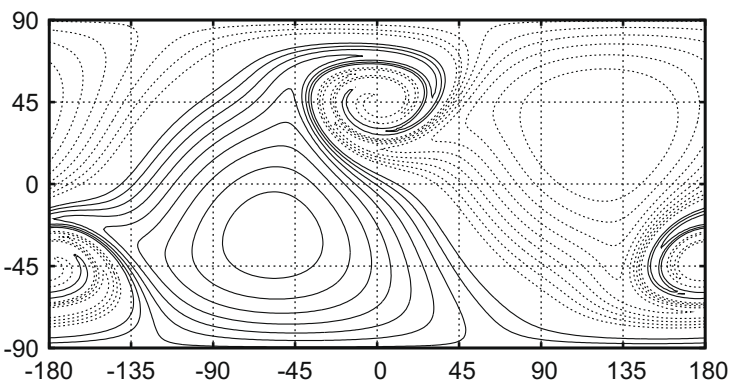

(c) day 9

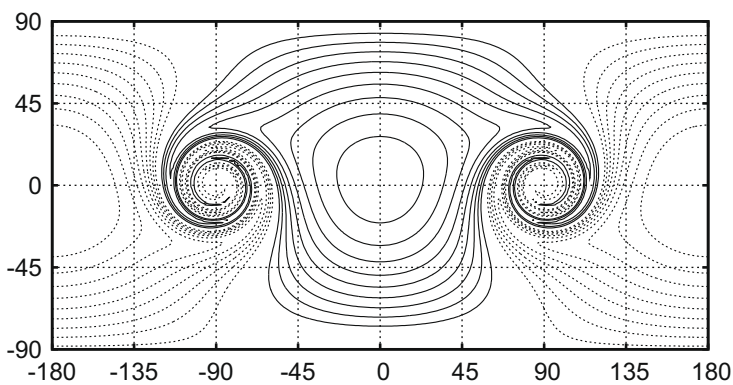

(e) exact at day 12

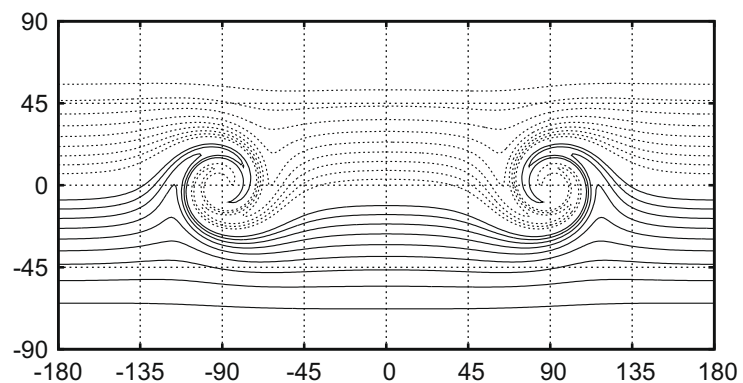

(b) day 6

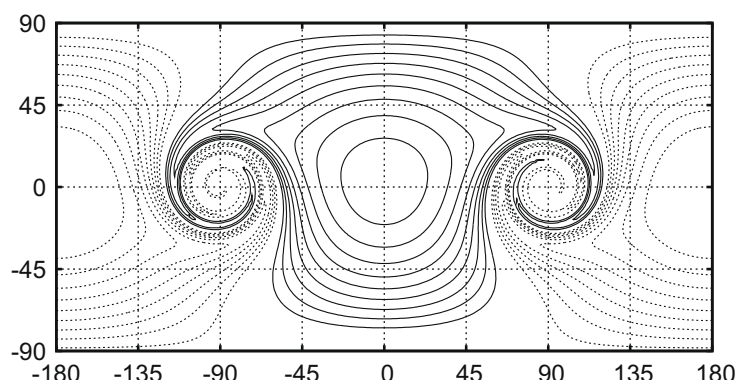

(d) day 12

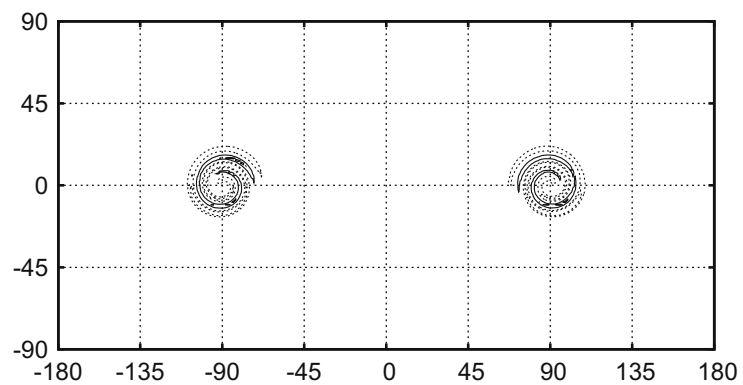

(f) difference at day 12

Fig. 8. Height fields of the moving vortex test computed by 3rd order MCV method on $N=30$ (DOFs = 54,002) at day 3, 6, 9 and 12 . Bottom figure shows exact solution and the difference with numerical result at day 12 . In panels (a)-(e), solid lines indicate the contours from 1.05 to 1.50 with 0.05 interval, and dash lines from 0.50 to 0.95 . In panel (f), solid lines stand for $0.02,0.04$ and 0.06 contours, while dash lines for -0.06 , -0.04 and -0.02 , respectively.

in the literature. The major vortical structure of the solution is accurately captured. The difference between the analytical solution and numerical one is also plotted in Fig. 8(f). The major error appears around the centers of the two vortices, where part of fine structure is under the grid resolution. The normalized error variations in time are plotted in Fig. 9 . The $L_{\infty}$ error is the largest one because of the local discontinuity, which is also reported in [32,33]. Our scheme is not sensitive to grid alignment even with this moving vortices velocity field.

\subsection{Global shallow water model}

We present in this part the major benchmark tests suggested in [58] for the global shallow water models. We also tested the model with a zonal geostrophic jet [13] to see the significance of the numerical error produced by grid which does not align with the jet flow.

\subsubsection{Williamson's test case 2: global steady zonal geostrophic flow [58]}

This is test case 2 in [58]. The initial velocity field is the same as (4.10), and the corresponding initial height in geostrophic balance is given by

$$
g h(\lambda, \theta, 0)=g h_{0}-\frac{u_{0}}{2}\left(2 a \Omega+u_{0}\right)(\sin \theta \cos \alpha-\cos \lambda \cos \theta \sin \alpha)^{2},
$$

where $u_{0}=2 \pi a /(12$ days $)$ and $g h_{0}=2.94 \times 10^{4}$. The Coriolis parameter in this test is given by 


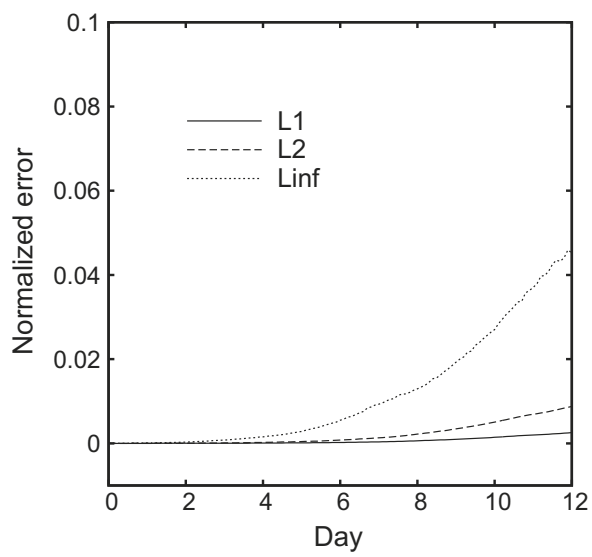

Fig. 9. Normalized errors of the 3rd order MCV method on $N=30$ (DOFs $=54,002$ ) in the moving vortex test on the sphere.

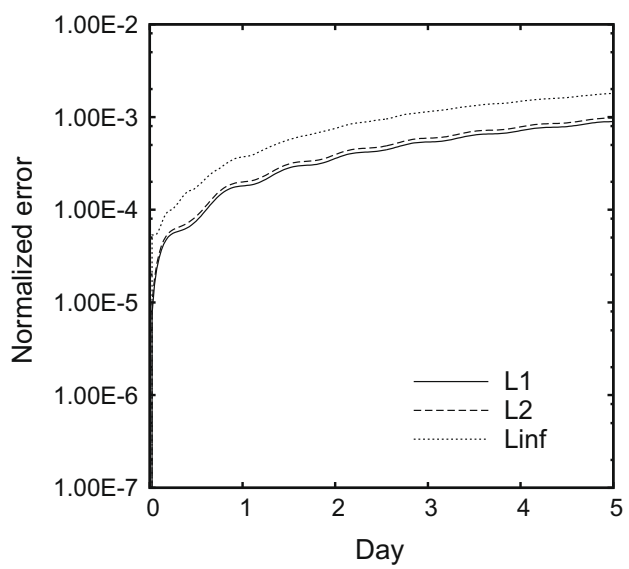

(a) 3rd order MCV on $N=25(\mathrm{DOFs}=37502)$

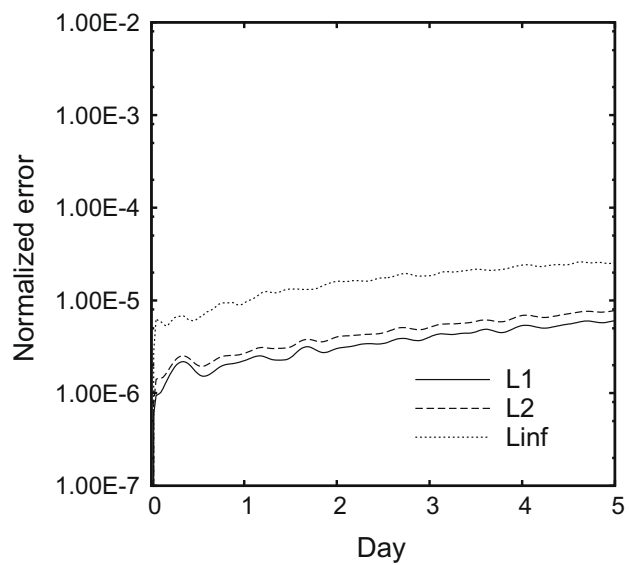

(b) 4th order MCV on $N=20(\mathrm{DOFs}=36002)$

Fig. 10. Normalized errors in test case 2 with $\alpha=\pi / 4$.

$$
f=2 \Omega(-\cos \lambda \cos \theta \sin \alpha+\sin \theta \cos \alpha) \text {. }
$$

In this test, the theoretical solution to this problem is a steady zonal flow maintaining the initial state in the balance between the geopotential gradient force and the Coriolis force.

We computed this test up to 5 days with a rotation angle being $\alpha=\pi / 4$ by both the third order scheme on $N=10$ mesh and the fourth order scheme on $N=8$ mesh.

The time evolution of the normalized errors for the height $h$ are plotted in Fig. 10. The results of the fourth order MCV method are much better than that of the third order one. The numerical convergence with $N=2,4,8,16$ meshes is shown in Table 2. Both third order and fourth order MCV methods achieve the expected orders of accuracy in the non-linear global shallow water model.

4.2.2. Williamson's test case 5: zonal flow over an isolated mountain [58]

The initial zonal flow for the total height $T=h+b$ is similar to those given in Section 4.2.1, but with the parameters $h_{0}=5960$ and $u_{0}=20$. In this test case, an isolated mountain defined as the bottom surface $b$ is centered at $\left(\lambda_{c}, \theta_{c}\right)=(3 / 2 \pi, \pi / 6)$, and the profile is given by

$$
b(\lambda, \theta)=b_{0}\left(1-\frac{\widehat{R}}{R}\right),
$$

where $b_{0}=2000, R=\pi / 9$, and $\widehat{R}=\min \left(R, \sqrt{\left(\lambda-\lambda_{c}\right)^{2}+\left(\theta-\theta_{c}\right)^{2}}\right)$. Thus, the initial depth of water is obtained by $h(\lambda, \theta, 0)=T(\lambda, \theta, 0)-b(\lambda, \theta)$. The computation was conducted with third order method on $N=25$ mesh and fourth order method on $N=20$ mesh. 
Table 2

Numerical error and convergence rate in test case 2 with the rotational angle $\alpha=\pi / 4$.

\begin{tabular}{|c|c|c|c|c|c|c|}
\hline$N$ & $L_{1}$ & Order & $L_{2}$ & Order & $L_{\infty}$ & Order \\
\hline \multicolumn{7}{|c|}{ 3rd order MCV } \\
\hline 2 & $5.51 \mathrm{E}-2$ & - & $6.03 E-2$ & - & $9.47 \mathrm{E}-2$ & - \\
\hline 4 & $1.20 \mathrm{E}-2$ & 2.20 & $1.29 \mathrm{E}-2$ & 2.22 & $2.10 \mathrm{E}-2$ & 2.17 \\
\hline 8 & $1.71 \mathrm{E}-3$ & 2.81 & $1.87 \mathrm{E}-3$ & 2.79 & $3.41 \mathrm{E}-3$ & 2.62 \\
\hline 16 & $2.23 \mathrm{E}-4$ & 2.94 & $2.46 \mathrm{E}-4$ & 2.93 & $4.51 \mathrm{E}-4$ & 2.92 \\
\hline \multicolumn{7}{|c|}{4 th order MCV } \\
\hline 2 & $6.77 \mathrm{E}-3$ & - & $8.39 E-3$ & - & $1.86 \mathrm{E}-2$ & - \\
\hline 4 & $2.95 \mathrm{E}-4$ & 4.52 & $3.72 \mathrm{E}-4$ & 4.50 & $9.76 \mathrm{E}-4$ & 4.25 \\
\hline 8 & $1.49 \mathrm{E}-5$ & 4.31 & $1.91 \mathrm{E}-5$ & 4.28 & $6.26 \mathrm{E}-5$ & 3.96 \\
\hline 16 & $8.95 E-7$ & 4.06 & $1.19 E-6$ & 4.00 & $4.38 \mathrm{E}-6$ & 3.84 \\
\hline
\end{tabular}

The total height after 15 days is shown in Fig. 11. The numerical results are quite similar to those of the spectral transform method with much higher resolution T213 (Fig. 5.1 of [5]). Smooth solutions around the mountain are obtained without unphysical oscillation.

We computed the conservation error $M(\psi)$ of the total mass (Mass), total energy (Ene) and the potential enstrophy (Ens) defined by

$$
\begin{aligned}
& \text { Mass }=h, \\
& \text { Ene }=\frac{1}{2}\left(g h^{2}+h \mathbf{u} \cdot \mathbf{u}\right), \\
& \text { Ens }=\frac{1}{2 h}(\zeta+f)^{2},
\end{aligned}
$$

where the vorticity $\zeta$ is computed by $\zeta=\mathbf{p} / a \cdot(\nabla \times \mathbf{u})$ with $\mathbf{p}=(x, y, z)$ denoting the position vector on the global surface. The time history of the conservation errors are shown in Fig. 12. The conservation of the total mass is fulfilled due to the finite volume formulation. The total energy and the potential enstrophy is also adequately conserved. Our results of the third and fourth order schemes are competitive to those by the spectral transform method with T63 resolution (see Fig. 5.4 of [22]). Moreover, when comparing the results between the third order MCV method on $N=25$ mesh (37,502 DOFs) and the fourth order MCV method on $N=20$ mesh (36,002 DOFs), we find again the result of the high order method is apparently superior even with a coarser mesh.

\subsubsection{Williamson's test case 6: Rossby-Haurwitz wave [58]}

This example is the zonal Rossby-Haurwitz wave. It is known that the intrinsic dynamic instability of the physics might prevent it from being a proper example for testing the long-term performance of a numerical model [51]. Nevertheless, the Rossby-Haurwitz wave still provides a good test bed for global middle-term simulations. The numerical results up to 14 days calculated by the spectral method on the T213 grid (see Fig. 5.5 of [5]) are widely accepted as the reference solutions that reasonably reflect the dynamic behavior of the system.

The details of the initial height and velocity fields are given in [58]. We computed the test by both third and fourth order schemes with different mesh resolutions.

The height field after 14 days are shown in Fig. 13. The results after 14 days are slightly different between third order method and fourth order method. Isolated centers of low geopotential height is observed in the result of the third order

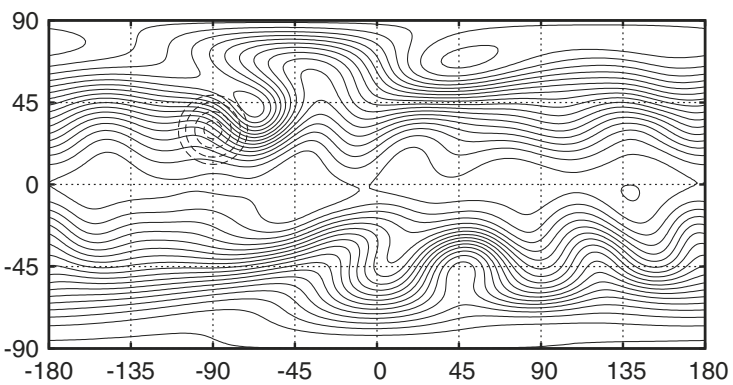

(a) 3rd order $\mathrm{MCV}$ on $N=25(\mathrm{DOFs}=37502)$

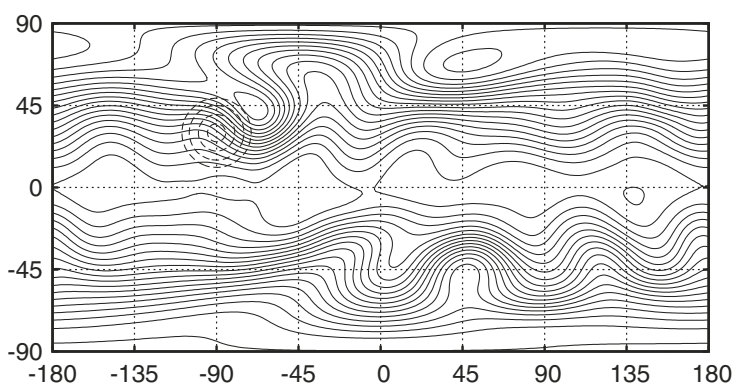

(b) 4th order MCV on $N=20(\mathrm{DOFs}=36002)$

Fig. 11. Total height field at day 15 for zonal flow over an isolated mountain. The contour interval is 50 from 5050 to 5950 . The dashed line is the mountain height with the contour value of 100, 500, 1000, 1500 and 2000. 


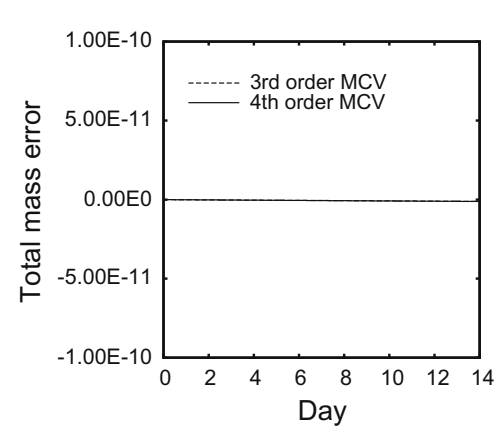

(a) Total mass

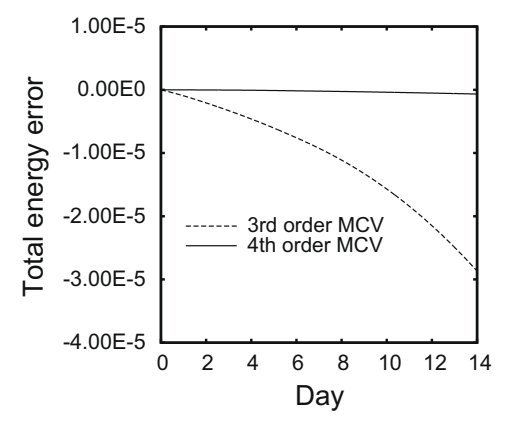

(b) Total energy

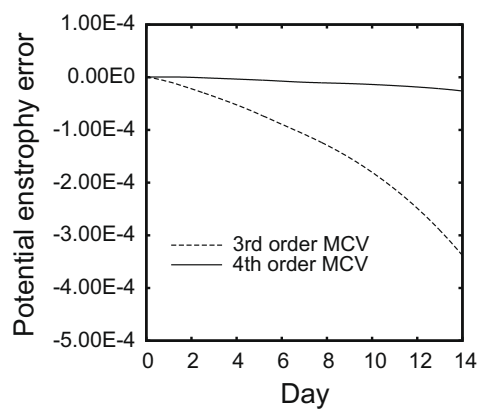

(c) Potential enstrophy

Fig. 12. Time evolution of the normalized conservation errors computed by the 3rd order MCV method on $N=25(\mathrm{DOFs}=37,502)$ and 4 th order $\mathrm{MCV}$ method on $N=20$ (DOFs $=36,002)$ in test case 5 .

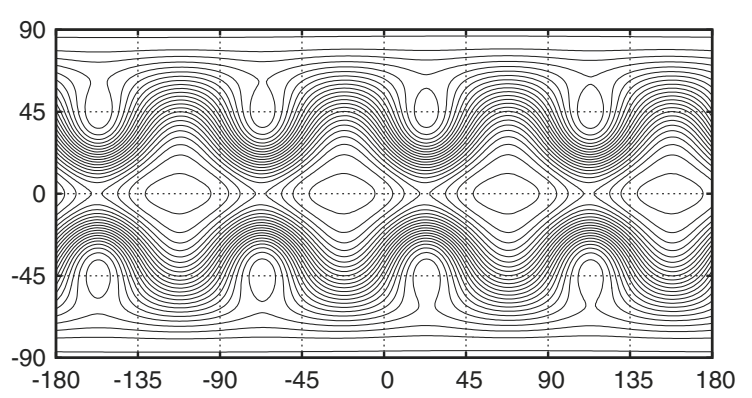

(a) 3rd order $\mathrm{MCV}$ on $N=40(\mathrm{DOFs}=96002)$

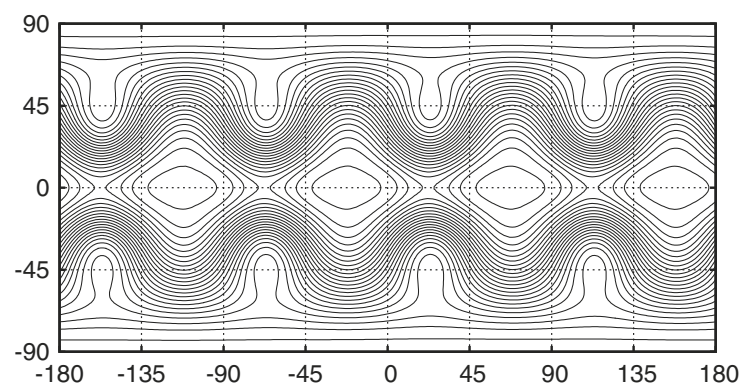

(b) 4th order MCV on $N=32(\mathrm{DOFs}=92162)$

Fig. 13. The height field at day 14 for the Rossby-Haurwitz wave. The contour interval is 100 from 8100 to 10,500 .

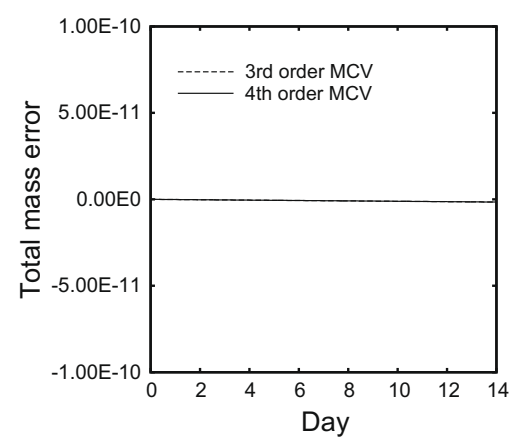

(a) Total mass

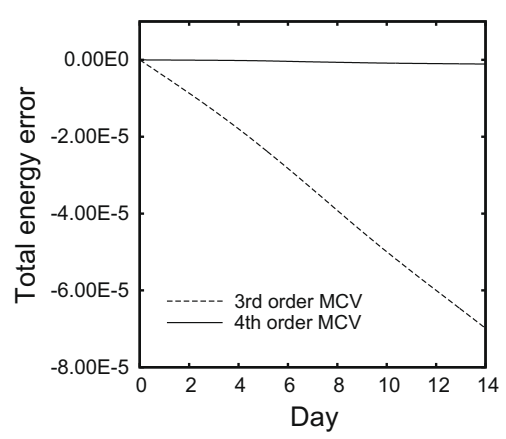

(b) Total energy

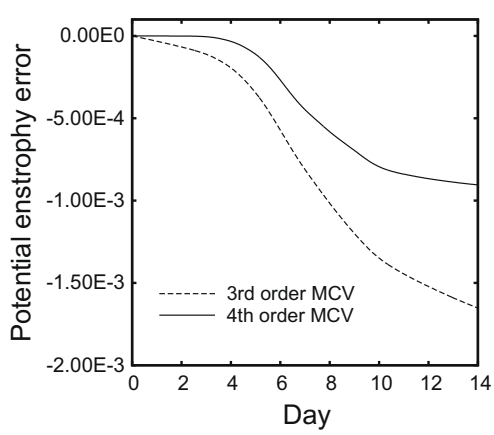

(c) Potential enstrophy

Fig. 14. Time evolution of the normalized conservation errors computed by the 3rd order MCV method on $N=40$ (DOFs $=96,002)$ and 4 th order MCV method on $N=32$ (DOFs $=92,162$ ) in test case 5 .

scheme. With improved accuracy of the scheme, the fourth order MCV method gives very similar solution to the reference one in [5].

We also plotted the time evolution of the normalized conservation errors for the total mass, total energy and potential enstrophy in Fig. 14. The same conclusion with respect to the performance of the third and fourth order schemes on different mesh resolutions can be drawn.

It is noted that with nearly the same DOFs the computational time of the third order MCV method on $N=40$ mesh is about 1.29 times of that taken by the fourth order MCV method on $N=32$ mesh.

\subsubsection{Perturbed flow in balanced jet field [13]}

The last example is the development of a barotropic instability induced by a small perturbation added to a geostrophically balanced jet flow (see [13] for detailed numerical configuration). This is a quite challenging problem for any grid model 


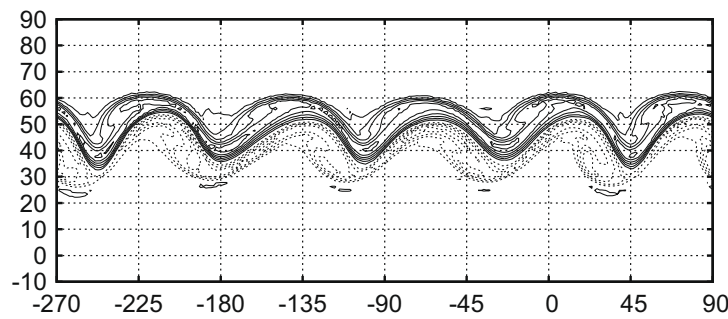

(a) $N=30(\mathrm{DOFs}=54002)$

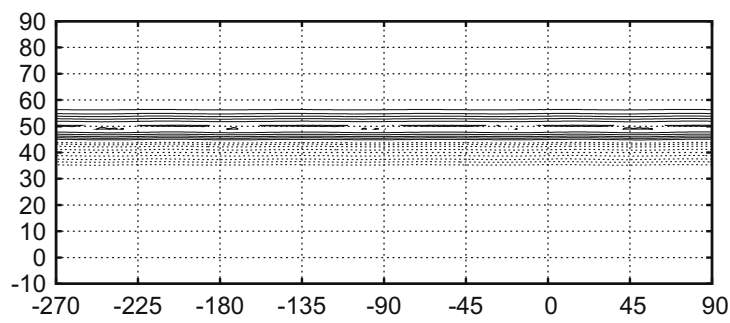

(b) $N=120($ DOFs $=864002)$

Fig. 15. Vorticity result of the 3rd order MCV method in balanced jet field without perturbation at day 5 .

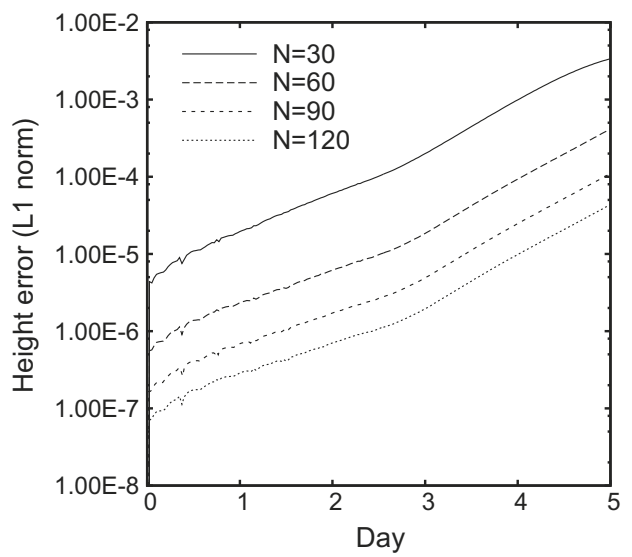

Fig. 16. Normalized error of the height without perturbation in the balanced jet flow computed by the 3rd order MCV method.

having its grid not aligned to the zonal direction. We first computed the undisturbed zonal jet flow until 5 days with the grid resolutions gradually refined as $N=30,60,90$ and 120 . As observed in other models using cubed-sphere grid and icosahedral grid, the solution could not rigorously maintain the initial zonal steady flow. Shown in Fig. 15, a disturbance of wavenumberfive is developed and clearly observed in the case with $N=30$. Nevertheless, a significant improvement can be observed when the grid resolution is refined to $N=120$. Moreover, the numerical oscillation in the MCV results looks much smaller than that of the high order DG method in [23]. We examined the $L_{1}$ errors of the height field in time and displayed in Fig. 16, which shows a uniform convergence.

Next, we computed the instability generated by the small perturbation until 6 days. The vorticity fields computed with $N=30,60,90$ and 120 resolutions are shown in Fig. 17. Again, a wavenumber-five pattern is visible with low grid resolution $(N=30)$. The results with higher grid resolutions, on the other hand, rapidly converge to the physical solution as those in $[13,4]$.

\subsection{Remarks on implementing the multi-moment methods on various spherical grids}

The multi-moment finite volume method has been implemented on the Yin-Yang grid [24] and cubed-sphere grid [4] previously by our group. As it is well known, both Yin-Yang grid and cubed-sphere grid use quadrilateral grid cell (element), and enjoy the advantages of the structured grid. In particular, the Yin-Yang grid is composed of two truncated longitude-latitude grids, thus the existing numerical formulations developed on a longitude-latitude grid can be directly transplanted to the Yin-Yang grid. However, the Yin-Yang overset grid has to use an overlapping region to communicate data between two component grids, where interpolation is involved and the numerical conservation is not guaranteed. This drawback might raise some problems for applications where the conservation is of great importance. The cubedsphere grid, on the other hand, has a well connected finite volume mesh over the whole globe by hanging together the mesh cells from two neighboring patches on the cube surface, thus has numerical conservation if a finite volume formulation is used.

The multi-moment method uses different moments to build local high order reconstruction over compact stencil (only a single cell is needed in nearly all cases). The multi-moment reconstruction is very flexible and beneficial when applied to the Yin-Yang grid and the cubed-sphere grid. The data transfer across the patch (or component grid) boundary can be conducted 


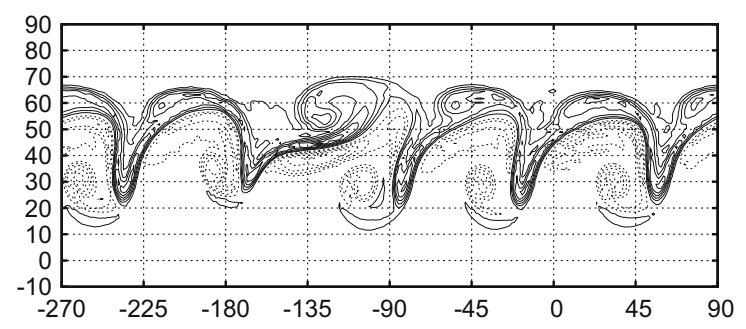

(a) $N=30(\mathrm{DOFs}=54002)$

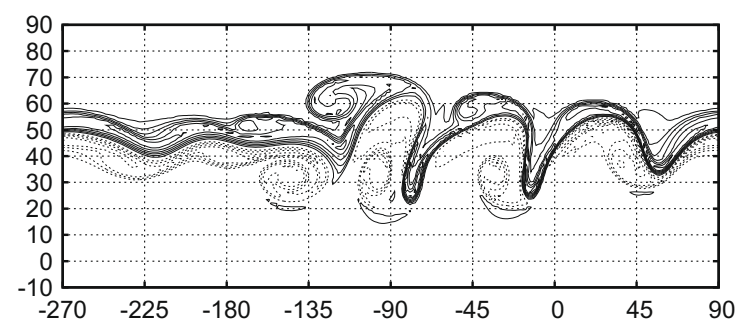

(b) $N=60(\mathrm{DOFs}=216002)$

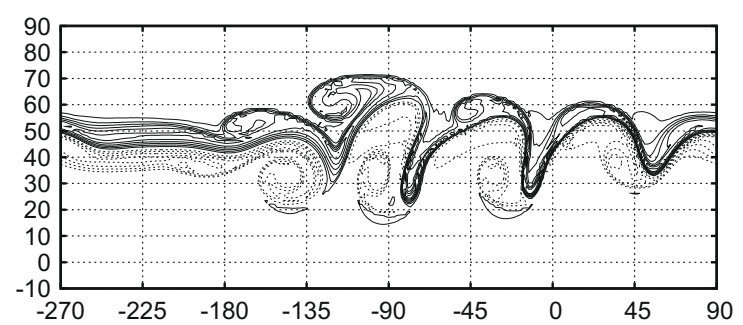

(c) $N=90(\mathrm{DOFs}=486002)$

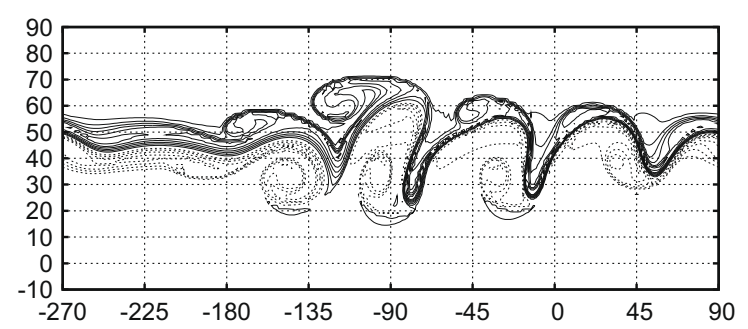

(d) $N=120($ DOFs $=864002)$

Fig. 17. Vorticity result of the 3rd order MCV method in balanced jet field with perturbation at day 6 .

by single-cell reconstruction on either side. This minimizes the halo layer of the Yin-Yang overset grid and thus reduces the conservative errors effectively. The performance of the multi-moment finite volume method in these two spherical grids are adequate and promising. We have not applied the MCV method [20] to the Yin-Yang grid and the cubed-sphere grid for the moment, but can expect the outcome to be as good as or better than the previous multi-moment finite volume method. As mentioned before, the MCV method does not explicitly involve the computation of cell-integrated quantity, and is more convenient when applied to real problems with complex geometry or source terms.

Compared to above two grids, the icosahedral grid is more geometrically isotropic and has the most uniform grid elements over the whole globe. Perhaps, the major barrier to this grid is the lack of high accurate and easy-to-use numerical schemes for its unstructured grid configuration. We hope the numerical formulations presented in this paper can provide a practical framework for the icosahedral grid. As the multi-moment method is still evolving, further exploration will be made for numerical cores of higher quality.

Before ending this section, we address that it is very easy to enhance the monotonicity in the multi-moment finite volume method, which might be important in computing the transport of a concentration. We introduce the first order derivative at the cell center as an additional constraint for the multi-moment reconstruction which works as a slope limiter and effectively suppresses the numerical oscillations in the solution, see $[60,65]$ among others for example. 


\section{Concluding remarks}

A novel accurate numerical framework for global shallow water equations has been developed using the high order multimoment constrained finite volume (MCV) method and the icosahedral geodesic grid. The multi-moment formulation shares the essential properties that are usually expected for computational fluid applications, such as the numerical conservation, high order accuracy, robustness and flexibility due to the local reconstruction, hence is well suited for the icosahedral geodesic grid. Moreover, the unknowns (computational variables) in the MCV method are the point values. There is no spatial integration involved in the numerical procedure. This is not only a great benefit to the computational efficiency but also advantageous (or essential to some extent) for the accurate treatments of the spherical geometry, e.g. the curved triangular cell element and the projection of the governing equations from the 3D Cartesian coordinate system onto the surface of the sphere, as well as the computation of the source terms.

Numerical examples presented in this paper reveal that the proposed model can achieve competitive outputs even with relatively lower mesh resolutions. We can expect the formulations presented in this paper to be a promising base for further development of global atmospheric and oceanic models.

\section{Appendix A. Icosahedral geodesic grid}

In this appendix, we describe how to generate the icosahedral geodesic grid based on an approach by Sadourny et al. [43]. The grid is generated from the regular icosahedron shown in Fig. 1. For simplicity, we assume that the radius of the circumscribed sphere is one and the center of the sphere at the origin $O$ of the Cartesian coordinate.

The 12 vertices of the regular icosahedron points in the Cartesian coordinate system are given as,

$$
\left\{\begin{array}{l}
\left(x_{p 1}, y_{p 1}, z_{p 1}\right)=(0,0,1), \\
\left(x_{p 2}, y_{p 2}, z_{p 2}\right)=(2 \hat{a}, 0, \hat{a}), \\
\left(x_{p 3}, y_{p 3}, z_{p 3}\right)=(\hat{b}, \hat{e}, \hat{a}), \\
\left(x_{p 4}, y_{p 4}, z_{p 4}\right)=(-\hat{c}, \hat{d}, \hat{a}), \\
\left(x_{p 5}, y_{p 5}, z_{p 5}\right)=(-\hat{c},-\hat{d}, \hat{a}), \\
\left(x_{p 6}, y_{p 6}, z_{p 6}\right)=(\hat{b},-\hat{e}, \hat{a}), \\
\left(x_{p 7}, y_{p 7}, z_{p 7}\right)=(0,0,-1), \\
\left(x_{p 8}, y_{p 8}, z_{p 8}\right)=(\hat{c}, \hat{d},-\hat{a}), \\
\left(x_{p 9}, y_{p 9}, z_{p 9}\right)=(-\hat{b}, \hat{e},-\hat{a}), \\
\left(x_{p 10}, y_{p 10}, z_{p 10}\right)=(-2 \hat{a}, 0,-\hat{a}), \\
\left(x_{p 11}, y_{p 11}, z_{p 11}\right)=(-\hat{b},-\hat{e},-\hat{a}), \\
\left(x_{p 12}, y_{p 12}, z_{p 12}\right)=(\hat{c},-\hat{d},-\hat{a}),
\end{array}\right.
$$

where

$$
\hat{a}=\frac{1}{\sqrt{5}}, \quad \hat{b}=\frac{1-\hat{a}}{2}, \quad \hat{c}=\frac{1+\hat{a}}{2}, \quad \hat{d}=\sqrt{\hat{b}} \quad \text { and } \quad \hat{e}=\sqrt{\hat{c}} .
$$

We coincide the north-pole of the sphere with $\left(x_{p 1}, y_{p 1}, z_{p 1}\right)$ and the south pole with $\left(x_{p 7}, y_{p 7}, z_{p 7}\right)$.

Let any triangle element $\triangle A B C$ of the icosahedron has its three vertices at $A=\left(x_{A}, y_{A}, z_{A}\right), B=\left(x_{B}, y_{B}, z_{B}\right)$ and $C=\left(x_{C}, y_{C}, z_{C}\right)$ (see Fig. 18). The grid is then created by dividing each edge of $\triangle A B C$ into $N$ equal arcs. The coordinate of points $P_{n}=\left(x_{n}, y_{n}, z_{n}\right)(n=2, \ldots, N)$ on boundary $A B$ can be obtained by solving linear system

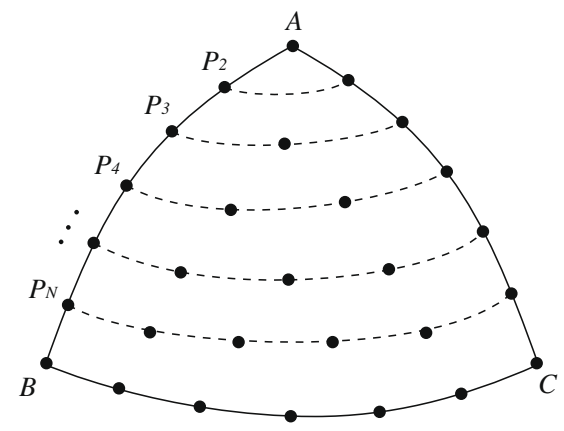

Fig. 18. Division of a piece of the icosahedron. 


$$
\begin{aligned}
& \left\{\begin{array}{l}
\overrightarrow{O A} \cdot \overrightarrow{O P}_{n}=\cos \left(\frac{n}{N} \angle(A B)\right), \\
\overrightarrow{O B} \cdot \overrightarrow{O P}_{n}=\cos \left(\left(1-\frac{n}{N}\right) \angle(A B)\right), \\
\overrightarrow{O P}_{n} \cdot(\overrightarrow{O A} \times \overrightarrow{O B})=0,
\end{array}\right. \\
& (n=2, \ldots, N),
\end{aligned}
$$

or in the Cartesian coordinate form,

$$
\begin{aligned}
& \left\{\begin{array}{l}
x_{A} x_{n}+y_{A} y_{n}+z_{A} z_{n}=\cos \left(\frac{n}{N} \angle(A B)\right), \\
x_{B} x_{n}+y_{B} y_{n}+z_{B} z_{n}=\cos \left(\left(1-\frac{n}{N}\right) \angle(A B)\right), \\
x_{n}\left(y_{A} z_{B}-z_{A} y_{B}\right)+y_{n}\left(z_{A} x_{B}-x_{A} z_{B}\right)+z_{n}\left(x_{A} y_{B}-y_{A} z_{B}\right)=0
\end{array}\right. \\
& (n=2, \ldots, N)
\end{aligned}
$$

where angle $\angle(A B)$ means arc $A B$ in radians. The third equation is a constraint to make $\overrightarrow{O A} \overrightarrow{O P}_{n}$ and $\overrightarrow{O B}_{\mathrm{O}}$ coplanar. In a similar manner, the grid points on edges $(B C)$ and $(A C)$, as well as the intersection points of the curves, can be found.

\section{References}

[1] R. Akoh, S. Ii, F. Xiao, A CIP/multi-moment finite volume method for shallow water equations with source terms, Int. J. Numer. Method Fluid 56 (2008) 2245-2270.

[2] P.-E. Bernald, J.-F. Remacle, R. Comblen, V. Legat, K. Hillewaert, High-order discontinuous Galerkin schemes on general 2D manifolds applied to the shallow water equations, J. Comput. Phys. 228 (2009) 6514-6535.

[3] A. Bermudez, M.E. Vazquez, Upwind methods for hyperbolic conservation laws with source terms, Comput. Fluid 23 (1994) 1049-1071.

[4] C.G. Chen, F. Xiao, Shallow water model on spherical-cubic grid by multi-moment finite volume method, J. Comput. Phys. 227 (2008) 5019-5044.

[5] R.J. Chien, J.J. Hack, D. Williamson, Spectral transform solutions to the shallow water test set, J. Comput. Phys. 119 (1995) $164-187$.

[6] J. Côté, A Lagrange multiplier approach for the metric terms of semi-Lagrangian models on the sphere, Quart. J. R. Meteorol. Soc. 114 (1988) 1347.

[7] B. Cockburn, C.-W. Shu, TVB Runge-Kutta local projection discontinuous Galerkin finite element method for conservation laws. II: General framework, Math. Comput. 52 (1989) 411-435.

[8] B. Cockburn, S.Y. Lin, C.-W. Shu, TVB Runge-Kutta local projection discontinuous Galerkin finite element method for conservation laws. III: Onedimensional systems, J. Comput. Phys. 84 (1989) 90-113.

[9] B. Cockburn, S. Hou, C.-W. Shu, TVB Runge-Kutta local projection discontinuous Galerkin finite element method for conservation laws. IV: The multidimensional case, Math. Comput. 54 (1990) 545-581.

[10] B. Cockburn, C.-W. Shu, The Runge-Kutta discontinuous Galerkin method for conservation laws. V: The multidimensional systems, J. Comput. Phys. 141 (1998) 199-224.

[11] R. Comblen, S. Legrand, E. Deleersnijder, V. Legat, A finite element method for solving the shallow water equations on the sphere, Ocean Model. 28 (2009) 12-23.

[12] C.A. Doswell III, A kinematic analysis of frontogenesis associated with a nondivergent vortex, J. Atmos. Sci. 41 (1984) $1242-1248$.

[13] J. Galewsky, R.K. Scott, L.M. Polvani, An initial-value problem for testing numerical models of the global shallow-water equations, Tellus 56A (2004) 429-440.

[14] F.X. Giraldo, A spectral element shallow water model on spherical geodesic grids, Int. J. Numer. Method Fluid 35 (2001) 869-901.

[15] F.X. Giraldo, J.S. Hesthaven, T. Warburton, Nodal high-order discontinuous Galerkin methods for the spherical shallow water equations, J. Comput. Phys. 181 (2002) 499-525.

[16] F.X. Giraldo, T. Warburton, A nodal triangle-based spectral element method for the shallow water equations on the sphere, J. Comput. Phys. 207 (2005) $129-150$.

[17] F.X. Giraldo, High-order triangle-based discontinuous Galerkin methods for hyperbolic equations on a rotating sphere, J. Comput. Phys. 214 (2006) 447-465.

[18] S. Ii, M. Shimuta, F. Xiao, A 4th-order and single-cell-based advection scheme on unstructured grids using multi-moments, Comput. Phys. Commun. 173 (2005) 17-33.

[19] S. Ii, F. Xiao, CIP/multi-moment finite volume method for Euler equations: a semi-Lagrangian characteristic formulation, J. Comput. Phys. 222 (2007) $849-871$.

[20] S. Ii, F. Xiao, A high order finite volume method using multi-moment constraints. Part I: Basic formulation, J. Comput. Phys. 228 (2009) 3669-3707.

[21] Y. Imai, T. Aoki, K. Takizawa, Conservative form of interpolated differential operator scheme for compressible and incompressible fluid dynamics, J. Comput. Phys. 227 (2008) 2263-2285.

[22] R. Jakob, J.J. Hack, D.L. Willamson, Solutions to the shallow water test set using the spectral transform method, Tech. Rep. NCAR/TN-388 + STR, National Center for Atmospheric Research (1993).

[23] M. Läuter, F.X. Giraldo, D. Handorf, K. Dethloff, A discontinuous Galerkin method for the shallow water equations in spherical triangular coordinates, J. Comput. Phys. 227 (2008) 10226-10242.

[24] X.L. Li, D.H. Chen, X.D. Peng, K. Takahashi, F. Xiao, A multi-moment finite volume shallow water model on Yin-Yang overset spherical grid, Mon. Weather Rev. 136 (2008) 3066-3086.

[25] D. Majewski, D. Liermann, P. Prohl, B. Ritter, M. Buchhold, T. Hanisch, G. Paul, W. Wergen, J. Baumgardner, The operational global icosahedralhexagonal gridpoint model GME: description and high-resolution tests, Mon. Weather Rev. 130 (2002) 319-338.

[26] Y. Masuda, H. Ohnishi, An integration scheme of the primitive equation model with an icosahedral-hexagonal grid system and its application to the shallow water equations, in: Short- and Medium-Range Numerical Weather Prediction, 1986, pp. 317-326.

[27] A. McDonald, Semi-Lagrangian advection on conformal-cubic grids, Mon. Weather Rev. 124 (1996) 1311-1322.

[28] A. McDonald, Semi-Lagrangian advection on a cubic gnomonic projection of the sphere, Atmos. Ocean. Memorial Volume (1997) 153-169.

[29] R.D. Nair, J. Côté, A. Staniforth, Cascade interpolation for semi-Lagrangian advection over the sphere, Quart. J. Roy. Meteorol. Soc. 125 (1999) 14451468.

[30] R.D. Nair, S.J. Thomas, R.D. Loft, A discontinuous Galerkin transport scheme on the cubed sphere, Mon. Weather Rev. 133 (2004) 814-828.

[31] R.D. Nair, S.J. Thomas, R.D. Loft, A discontinuous Galerkin global shallow water model, Mon. Weather Rev. 133 (2004) $876-888$.

[32] R.D. Nair, C. Jablonowski, Moving vortices on the sphere: a test case for horizontal advection problems, Mon. Weather Rev. 136 (2008) 699-711.

[33] W.M. Putman, S.-J. Lin, Finite-volume transport on various cubed-sphere grids, J. Comput. Phys. 227 (2007) 55-78.

[34] J. Qiu, C.-W. Shu, Hermite WENO schemes and their application as limiters for Runge-Kutta discontinuous Galerkin method: one-dimensional case, J. Comput. Phys. 193 (2003) 115-135. 
[35] J. Qiu, C.-W. Shu, Hermite WENO schemes and their application as limiters for Runge-Kutta discontinuous Galerkin method. II: Two-dimensional case, Comput Fluid 34 (2005) 642-663.

[36] M. Rancic, R.J. Purser, F. Mesinger, A global shallow-water model using an expanded spherical cube: gnomonic versus conformal coordinates, Quart. J. R. Meteorol. Soc. 122 (1996) 959-982.

[37] W.H. Reed, T.R. Hill, Triangular mesh methods for the Neutron transport equation, Technical Report LA-UR-73-479, Los Alamos Scientific Laboratory Report (1973).

[38] T.D. Ringler, R.P. Heikes, D.A. Randall, Modeling the atmospheric general circulation using a spherical geodesic grid: a new class of dynamical cores, Mon. Weather Rev. 128 (2000) 2471-2490.

[39] D.A. Randall, T.D. Ringler, R.P. Heikes, P. Jones, J. Baumgardner, Climate modeling with spherical geodesic grids, Comput. Sci. Eng. 4 (2002) $32-41$.

[40] P.L. Roe, Approximate Riemann solvers, parameter vectors and difference schemes, J. Comput. Phys. 43 (1981) 357-372.

[41] C. Ronchi, R. Iacono, P.S. Paolucci, The "cubed Spher": a new method for the solution of partial differential equations in spherical geometry, J. Comput. Phys. 124 (1996) 93-114.

[42] J.A. Rossmanith, A wave propagation method for hyperbolic systems on the sphere, J. Comput. Phys. 213 (2006) $629-658$.

[43] R. Sadourny, A. Arakawa, Y. Mintz, Integration of the nondivergent barotropic vorticity equation with an icosahedral hexagonal grid for the sphere, Mon. Weather Rev. 96 (1968) 351-356.

[44] R. Sadourny, Conservative finite-difference approximations of the primitive equations on quasi-uniform spherical grids, Mon. Weather Rev. 100 (1972) $136-144$.

[45] M. Satoh, T. Matsuno, H. Tomita, H. Miura, T. Nasuno, S. Iga, Nonhydrostatic icosahedral atmospheric model (NICAM) for global cloud resolving simulations, J. Comput. Phys. 227 (2008) 3486-3514.

[46] C.-W. Shu, Total-variation-diminishing time discretizations, SIAM J. Sci. Stat. Comput. 9 (1988) 1073-1084.

[47] C.G. Song, J.P. Wolf, The scaled boundary finite element method-alias consistent infinitesimal finite element cell method-for diffusion, Int. J. Numer. Method Eng. 45 (1999) 1403-1431.

[48] G.R. Stuhne, W.R. Peltier, New icosahedral grid-point discretizations of the shallow water equations on the sphere, J. Comput. Phys. 148 (1999) $23-58$.

[49] G.R. Stuhne, W.R. Peltier, A robust unstructured grid discretization for 3-dimensional hydrostatic flows in spherical geometry: a new numerical structure for ocean general circulation modeling, J. Comput. Phys. 213 (2006) 704-729.

[50] P.N. Swarztrauber, D.L. Williamson, J.B. Drake, The Cartesian method for solving partial differential equations in spherical geometry, Dynam. Atmos. Ocean 27 (1997) 679-706.

[51] J. Thuburn, Y. Li, Numerical simulations of Rossby-Haurwitz waves, Tellus 52A (2000) 181-189.

[52] V.A. Titarev, E.F. Toro, ADER: arbitrary high order Godunov approach, J. Sci. Comput. 17 (2002) 609-618.

[53] E.F. Toro, R.C. Millington, L.A.M. Nejad, Towards very high order Godunov schemes, in: E.F. Toro (Ed.), Godunov Methods: Theory and Applications, Kluwer Academic, 2001, pp. 907-940.

[54] H. Tomita, M. Tshugawa, M. Satoh, K. Goto, Shallow water model on a modified icosahedral geodesic grid by using spring dynamics, J. Comput. Phys. 174 (2001) 579-613.

[55] H. Tomita, M. Satoh, K. Goto, An optimization of the icosahedral grid modified by spring dynamics, J. Comput. Phys. 183 (2002) 307-331.

[56] D.L. Williamson, Integration of the barotropic vorticity equation on a spherical geodesic grid, Tellus 20 (1968) 642-653.

[57] D.L. Williamson, Integration of the primitive barotropic model over a spherical geodesic grid, Mon. Weather Rev. 95 (1969) $512-520$.

[58] D.L. Williamson, J.B. Drake, J.J. Hack, Rudiger Jakob, P.N. Swarztrauber, A standard test set for numerical approximations to the shallow water equations on the sphere, J. Comput. Phys. 101 (1992) 211-224.

[59] D.L. Williamson, The evolution of dynamical cores for global atmospheric models, J. Meteorol. Soc. Jpn. 85B (2007) 241-269.

[60] F. Xiao, T. Yabe, Completely conservative and oscillation-less semi-Lagrangian schemes for advection transportation, J. Comput. Phys. 170 (2001) 498.

[61] F. Xiao, Unified formulation for compressible and incompressible flows by using multi-integrated moment method: one-dimensional inviscid compressible flow, J. Comput. Phys. 195 (2004) 629-654.

[62] F. Xiao, A. Ikebata, T. Hasegawa, Numerical simulations of free-interface fluids by a multi-integrated moment method, Comput. Struct. 83 (2005) 409423.

[63] F. Xiao, R. Akoh, S. Ii, Unified formulation for compressible and incompressible flows by using multi-integrated moments. II: Multi-dimensional version for compressible and incompressible flows, J. Comput. Phys. 213 (2006) 31-56.

[64] F. Xiao, S. Ii, CIP/multi-moment finite volume method with arbitrary order of accuracy, JSCES J. 12 (2007) 873.

[65] F. Xiao, T. Okazaki, M. Satoh, An accurate semi-Lagrangian scheme for rain drop sedimentation, Mon. Weather Rev. 131 (2003) $810-819$.

[66] Y.L. Xing, C.W. Shu, High order finite difference WENO schemes with the exact conservation property for the shallow water equations, J. Comput. Phys. 208 (2005) 206-227.

[67] T. Yabe, T. Aoki, A universal solver for hyperbolic-equations by cubic-polynomial interpolation. 1 . One-dimensional solver, Comput. Phys. Commun. 66 (1991) 219-232.

[68] T. Yabe, F. Xiao, T. Utsumi, The constrained interpolation profile method for multiphase analysis, J. Comput. Phys. 169 (2001) $556-593$.

[69] M.P. Zhang, C.W. Shu, An analysis of and a comparison between the discontinuous Galerkin and the spectral finite volume methods, Comput. Fluid 34 (2005) 581-592. 